\title{
TOPOLOGICAL APPLICATIONS OF GENERIC HUGE EMBEDDINGS
}

\author{
FRANKLIN D. TALL
}

\begin{abstract}
In the Foreman-Laver model obtained by huge cardinal collapse, for many $\Phi, \Phi\left(\aleph_{1}\right)$ implies $\Phi\left(\aleph_{2}\right)$. There are a variety of set-theoretic and topological applications, in particular to paracompactness. The key tools are generic huge embeddings and preservation via $\kappa$-centred forcing. We also formulate "potent axioms" à la Foreman which enable us to transfer from $\aleph_{1}$ to all cardinals. One such axiom implies that all $\aleph_{1}$-collectionwise normal Moore spaces are metrizable. It also implies (as does Martin's Maximum) that a first countable generalized ordered space is hereditarily paracompact iff every subspace of size $\aleph_{1}$ is paracompact.
\end{abstract}

Assuming the consistency of a huge cardinal, Foreman and Laver [FL] construct a model of ZFC + GCH in which (as they put it) $\aleph_{2}$ possesses some "downwards transfer properties". For example, in their model every graph of size and chromatic number $\aleph_{2}$ has a vertex-induced subgraph of size and chromatic number $\aleph_{1}$, and groups of size $\aleph_{2}$ are the union of $\aleph_{1}$ free subgroups if all their subgroups of size $\leq \aleph_{1}$ are free. We shall establish in their model a variety of such transfer propositions in a topological context. Actually, we think of taking properties holding at $\aleph_{1}$ and transferring them to $\aleph_{2}$. The method of proof is to use an elementary embedding with strong closure properties definable in an $\aleph_{1}$-centred $* \aleph_{2}$-closed forcing extension of the model to perform the transfer, and then argue that the forcing could not have accomplished the transfer unless it already held. It is natural to generalize this idea to transfer from $\aleph_{1}$ to other cardinals. It is not known at present how to accomplish this from the usual large cardinals, so we proceed axiomatically in the style of $\left[\mathrm{Fo}_{3}\right]$. Our results impinge on a number of areas of set-theoretic topology, but the most interesting ones deal with paracompactness, e.g. the one mentioned in the abstract. The techniques however are widely applicable, as can be seen not only from our results on trees and ideals, but from those of Foreman and Laver quoted above.

The paper is organized as follows. We state the basic features of the ForemanLaver model and use them to go from $\aleph_{1}$ to $\aleph_{2}$. Next we present a variety of counterexamples to show our results do not hold in ZFC. We then introduce axioms that enable us to transfer from $\aleph_{1}$ to arbitrary cardinals. We finish with some results proved from supercompact cardinals, and then a list of problems.

Received by the editors June 15, 1987.

1991 Mathematics Subject Classification. Primary 03E35, 03E55, 03E65, 54A35, 54D15, 54D20, 54F05; Secondary 54E30, 54E35.

The author acknowledges support from Grant A-7354 of the Natural Sciences and Engineering Research Council of Canada. 
We assume ability to follow simple forcing arguments. The proofs will be mysterious to those who have not seen elementary embeddings previously. $\left[\mathrm{T}_{5}\right.$, SRK and $\mathrm{DTW}_{1}$ ] may help. The problems discussed are topologically motivated, but not too much topological knowledge is assumed. Whatever is necessary may be found in [E]. However we shall put in more details than if we were writing solely for topologists. $\left[\mathrm{T}_{4}\right]$ may provide some motivation. Our notation is reasonably standard; we use " $P$ " for the universe of a partially ordered set $\mathscr{P}$, while the symbol " $\|$ " means "decides", as in " $p \| \Phi$ ".

I am indebted to F. Franek, M. Foreman, R. Laver, M. Magidor, and W. H. Woodin for conversations concerning huge cardinals. If this paper is at all readable, credit is due to the referee, who spotted numerous infelicitous phrasings and outright falsehoods in the previous version.

\section{The Foreman-LAVER MODEL}

Before plunging into the set theory, let me mention the sorts of results we shall obtain. First, some definitions.

Definition. A subset $Q$ of a partially ordered set $\mathscr{P}$ is $\delta$-centred ( $\delta$ a cardinal) if there is an $f: Q \rightarrow \delta$ such that for each $\alpha \in \delta$, any finite subset of $f^{-1}(\{\alpha\})$ has a lower bound in $\mathscr{P}$. Alternatively, if $Q=\bigcup_{\alpha<\delta} Q_{\alpha}$, each $Q_{\alpha}$ centred in $\mathscr{P}$.

Definition. Let $\alpha$ be an ordinal. An $\aleph_{\alpha}$-tree is a tree of height and cardinality $\aleph_{\alpha}$. An $\aleph_{\alpha}$-subtree of a tree $T$ is a subtree which is an $\aleph_{\alpha}$-tree. An $\aleph_{\alpha+1}$-tree $T$ is special if there is a function (a specializing function) $f: T \rightarrow \aleph_{\alpha}$ such that for all $s, t, u \in T$, if $s \leq t, u$ and $f(s)=f(t)=f(u)$, then $t$ and $u$ are comparable.

Definition. A collection $\mathscr{Y}$ of sets in a topological space is discrete if each point in the space has a neighbourhood meeting at most one member of the collection. A discrete collection $\mathscr{Y}$ is separated if there exist open sets $\left\{U_{Y}: Y \in \mathscr{Y}\right\}$ such that $Y \subseteq U_{Y}$, and if $Y \neq Y^{\prime}$ then $U_{Y} \cap U_{Y^{\prime}}=\varnothing$. A space $X$ is $\kappa-\lambda$ collectionwise normal if each discrete collection of $\leq \kappa$ sets, each of cardinality $\leq \lambda$, is separated. $X$ is $\kappa$-collectionwise normal if it is $\kappa$ - $\lambda$-collectionwise normal for all $\lambda . X$ is $\kappa$-collectionwise Hausdorff if it is $\kappa$-1-collectionwise normal. $X$ is weakly $\kappa$-collectionwise Hausdorff if each discrete collection of $\kappa$ points includes a separated subcollection of size $\kappa . X$ is collectionwise normal if it is $\kappa$-collectionwise normal for all $\kappa$. Similarly define collectionwise Hausdorff and weakly collectionwise Hausdorff.

Definition. A space is $\kappa$-paracompact ( $\kappa$-metacompact) if every open cover of size $\leq \kappa$ has a locally (point-)finite open refinement. (We use countably instead of " $\aleph_{0^{-}}$"). A space is $\kappa$ - $\lambda$-screenable if every open cover of size $\leq \kappa$ has an open refinement which is the union of $\leq \lambda$ collections, each of which is disjoint. Such a refinement is said to be $\lambda$-disjoint. A space is $\kappa$-screenable if it is $\kappa$ - $\aleph$ screenable. A space is screenable if it is $\kappa$ - $\aleph_{0}$-screenable for all $\kappa$.

Definition. $X$ is locally $\kappa$-c.c. if each point has a neighbourhood that does not include a disjoint open collection of size $\kappa . X$ has local density $\leq \kappa$ if each point has an open neighbourhood with a dense set of cardinality $\leq \kappa$. 
Theorem 1. In the Foreman-Laver model,

(a) If $T$ is an $\aleph_{2}$-tree with each $\aleph_{1}$-subtree special, then $T$ is special.

(b) If $X$ is first countable and weakly $\aleph_{1}$-collectionwise Hausdorff, then it is weakly $\aleph_{2}$-collectionwise Hausdorff.

(c) If $X$ is first countable, $\aleph_{1}$-collectionwise Hausdorff and locally $\aleph_{2}$-c.c., then $X$ is $\aleph_{2}$-collectionwise Hausdorff.

(d) If $X$ is first countable, normal, and hereditarily $\aleph_{1}$-paracompact, then if $|X| \leq \aleph_{2}, X$ is paracompact.

(e) If $X$ is first countable and for every open $Y \subseteq X, Y \times\left(\omega_{1}+1\right)$ is normal, then if $|X| \leq \aleph_{2}, X$ is paracompact.

(f) If $X$ is first countable, normal, and $\aleph_{1}$-paracompact, then $X$ is $\aleph_{2}-\aleph_{2}$ collectionwise normal.

(g) If $X$ is an $\aleph_{1}$-collectionwise normal Moore space, $|X| \leq \aleph_{2}$, then $X$ is metrizable.

Now for the set theory.

Definition. An injection from a model $V$ into a model $M$ (of the same language) is an elementary embedding if for each $n$, for every formula $\Phi$ with $n$ free variables, and any $n$ elements $x_{1}, \ldots, x_{n} \in V, V \vDash \Phi\left[x_{1}, \ldots, x_{n}\right]$ if and only if $M \vDash \Phi\left[j\left(x_{1}\right), \ldots, j\left(x_{n}\right)\right]$.

Definition. A cardinal $\kappa$ is huge if there exists an elementary embedding $j: V$ $\rightarrow M$, where $M$ is a transitive subclass of $V$, the universe of sets, such that $j \mid \kappa$ is the identity, $j(\kappa)=\lambda>\kappa$, and $M$ is closed under $j(\kappa)$-sequences. $\kappa$ is called the critical point of $j$.

For basic information about huge cardinals, see [SRK and $\mathrm{K}_{1}$ ]. Starting with a huge cardinal $\kappa, j(\kappa)=\lambda$, Foreman and Laver construct a partial order $\mathscr{P} * \mathscr{\mathscr { R }}$ such that $\mathscr{P}$ is $\kappa$-c.c., $V\left[G_{\mathscr{D}}\right] \vDash \kappa=\aleph_{1}, \dot{\mathscr{R}} \in V\left[G_{\mathscr{P}}\right]$ is $\lambda$-c.c. and countably (i.e. $\kappa$-)closed, and $V\left[G_{\mathscr{P}_{*} \mathscr{\mathscr { R }}}\right] \vDash \lambda=\aleph_{2} . \quad V\left[G_{\mathscr{P}_{*} \mathscr{\mathscr { R }}}\right]$ also satisfies GCH. $\mathscr{P} * \mathscr{\mathscr { R }}$ is constructed so that there is a complete embedding $h: \mathscr{P} * \mathscr{\mathscr { R }}$ into $j(\mathscr{P})$ such that:

(i) if $G$ is $\left(\mathscr{P}_{*} \dot{\mathscr{R}}\right)$-generic, then in $V[G], j(\mathscr{P}) / h^{\prime \prime} G$ is $\kappa\left(=\aleph_{1}\right)$-centred,

(ii) the embedding $j$ extends in $V\left[G_{j(\mathscr{P}) * j(\mathscr{R})}\right]$ to an elementary embedding (which we shall also call) $j: V\left[G_{\mathscr{P} * \mathscr{\mathscr { R }}}\right] \rightarrow M\left[G_{j(\mathscr{P}) * j(\dot{\mathscr{R}})}\right]$ which sends $\aleph_{1}^{V\left[G_{\mathscr{P} * \mathfrak{k}}\right]}$ to $\aleph_{2}^{V\left[G_{\mathscr{P} * \mathfrak{t}}\right]}$,

(iii) $M\left[G_{j(\mathscr{P}) * j(\dot{\mathscr{R}})}\right]$ is closed under $\lambda$-sequences of elements with names in $M$.

Note that the forcing to get from $V\left[G_{\mathscr{P}_{*} \dot{\mathscr{R}}}\right]$ to $V\left[G_{j(\mathscr{P}) * j(\dot{\mathscr{R}})}\right]$ is $\kappa$-centred followed by $\lambda$-closed, i.e. in the sense of $V\left[G_{\mathscr{P} * \mathscr{R}}\right], \aleph_{1}$-centered followed by $\aleph_{2}$-closed. Contrast this with the Lévy collapse with countable conditions of a weak compact or supercompact to $\aleph_{2}$. There we ask what properties of the cardinal $\lambda$ that becomes $\aleph_{2}$ are preserved by countably closed forcing; here we ask about $\aleph_{1}$-centred and $\lambda$-closed. $\lambda$-closed forcing preserves many properties of $\lambda$ (the $\Pi_{1}^{1}$ ones) so in fact the essential difference between the two collapses is "countably closed" versus " $\aleph_{1}$-centred". Neither is obviously better than the other; for that matter, it is very much an open question for set-theorists these days to get interesting consequences of huge cardinals that provably cannot be 
obtained from supercompacts. (Foreman (unpublished) does have some Chang Conjecture type results that yield inner models of hugeness.) As with the results of Foreman-Laver, we cannot obtain our results from supercompacts but neither can we prove that that cannot be done.

Let us prove (a) of Theorem 1. Let $V\left[G_{\mathscr{P}_{*} \mathscr{R}}\right] \vDash$ “ $T$ is an $\aleph_{2}$-tree with every $\aleph_{1}$-subtree special". Then $M\left[G_{j(\mathscr{P}) * j(\dot{R})}\right] \vDash " j(T)$ is a tree such that every $j\left(\aleph_{1}\right)$-subtree is special". By (iii) above, $j^{\prime \prime} T \in M\left[G_{j(\mathscr{P}) * j(\dot{\mathscr{R}})}\right] \cdot j^{\prime \prime} T$ is a $j\left(\aleph_{1}\right)=\aleph_{2}$-subtree of $j(T)$, so it is special. "Special" is upward absolute so $V\left[G_{j(\mathscr{P}) * j(\dot{R})}\right] \vdash j^{\prime \prime} T$ is special. $j^{\prime \prime} T$ is isomorphic to $T$ so $V\left[G_{j(\mathscr{P}) * j(\dot{R})}\right] \vDash$ " $T$ is special".

We now pull specialty back to $V\left[G_{\mathscr{P}_{*} \dot{R}}\right]$ in two stages.

Lemma 2. If $\mathscr{S}$ is a $\lambda$-closed partial order and $T$ is a $\lambda$-tree such that 1 ⿰冫一⿻上丨 " $\check{T}$ is special", then $T$ is special.

Proof. Let $T=\left\{t_{\alpha}\right\}_{\alpha<\lambda}$. Let $1 \Vdash$ “ $\dot{f}: \check{T} \rightarrow \check{\lambda}$ is a specializing function". Pick

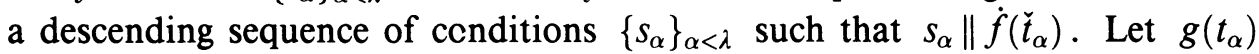
be that $\beta$ such that $s_{\alpha} \Vdash \dot{f}\left(\check{t}_{\alpha}\right)=\check{\beta}$. Claim $g$ specializes $T$, for if $t_{\alpha} \leq t_{\gamma}, t_{\delta}$ and $g\left(t_{\alpha}\right)=g\left(t_{\gamma}\right)=g\left(t_{\delta}\right)$, let $\eta=\max (\alpha, \gamma, \delta)$. Then $s_{\eta} \Vdash “ \check{t}_{\gamma}$ and $\check{t}_{\delta}$ are compatible". But then they are compatible.

Lemma 3. Suppose $P$ is $\kappa$-centred, $\kappa \leq \lambda, T$ is a $\lambda$-tree, and $1 \Vdash \mathscr{P}$ “ $\check{T}$ is special". Then $T$ is special.

Proof. Let $h: P \rightarrow \kappa$ witness $\kappa$-centering. Let $1 \Vdash$ " $\dot{f}: \check{T} \rightarrow \check{\lambda}$ is a specializing function". For each $t \in T$, pick $p_{t} \| \dot{f}(\check{t})$. Define $g: T \rightarrow \kappa \times \lambda$ by $g(t)=$ $\langle\eta, \xi\rangle$ iff $\eta$ is least such that $h\left(p_{t}\right)=\eta$, and $p_{t} \Vdash \dot{f}(\check{t})=\check{\xi}$. Then $g$ specializes $T$ except for the minor detail that its range is $\kappa \times \lambda$. But since only the cardinality of the range matters, that is O.K.

When one attempts to apply the technique we have just used for trees to topology, there is an added complication which we shall now discuss. In the tree-or more generally-partial order context, $j^{\prime \prime} P$ is not only a subset of $j(P)$ but also the order it inherits from $j(P)$ is the same as the one induced on it by $j^{\prime \prime}$, i.e. $\{\langle j(p), j(q)\rangle: p \leq q\}$. The situation is different in the topological context-the copy $\left\langle j^{\prime \prime} X,\left\{j^{\prime \prime} U: U \in \mathscr{T}\right\}\right\rangle$ of $\langle X, \mathscr{T}\rangle$ is not necessarily a subspace of $\langle j(X), j(\mathscr{T})\rangle$. We give examples of this in [DTW 1 and DTW $]$ and establish conditions under which it is a subspace. The most useful one is that the character of each point (least cardinal of a neighbourhood base at that point) is less than $\kappa$, the critical point of the embedding. We write " $\hat{\chi} \leq \kappa$ " to express this. If $\kappa=\aleph_{1}$, which is the case we are interested in in this paper, this reduces to requiring that $X$ be first countable (all points have countable character). There is an additional technical obstacle to the idea of getting a copy of $\langle X, \mathscr{T}\rangle$ to be a subspace of $\langle j(X), j(\mathscr{T})\rangle$ : we cannot a priori talk about $\left\{j^{\prime \prime} U: U \in \mathscr{T}\right\}$ in $M$ even if $X$ is first countable and $|X| \leq j(\kappa)$, since $|\mathscr{T}|$ is likely greater than $j(\kappa)$. The remedy is to instead, by first countability, fix a basis $\mathscr{B}$ for $X$ of cardinality $\leq|X|$ and note that the topology on $j^{\prime \prime} X$ generated by $\left\{j^{\prime \prime} B: B \in \mathscr{B}\right\}$ in $M$ is a member of $M$. Thus, in what follows, we will consider topological spaces $\langle X, \mathscr{B}\rangle$ (rather than $\langle X, \mathscr{T}\rangle$ ), where $\mathscr{B}$ is a basis for a topology on $X$. This has the additional advantage that we can use the single notation $\langle X, \mathscr{B}\rangle$ to denote whatever topology $\mathscr{B}$ generates on $X$ in various models. 
Fleissner $\left[F_{2}\right]$ has asked whether it is consistent-assuming large cardinalsthat first countable $\aleph_{1}$-collectionwise Hausdorff spaces are collectionwise Hausdorff. We cannot settle this but we solve related problems. Again, we proceed downwards in two steps.

Lemma 4. Suppose $|Y|=\lambda$ and some subset of $Y$ of cardinality $\lambda$ is separated after $\lambda$-closed forcing. Then there is such a subset in the ground model.

Proof. This is routine. Note that if a set is separated, there exist basic open sets separating it. Thus let $f: \lambda \rightarrow Y \times \mathscr{B}$ be a one-one function coding a separation in the extension, and pick a descending sequence of forcing conditions deciding $f$. The resulting function separates the first coordinates of its range.

Lemma 5. Suppose $\kappa<\operatorname{cf}(\lambda), \mathscr{P}$ is $\kappa$-centred, $|Y|=\lambda$, and $1 \Vdash_{P} \dot{f}: \check{\lambda} \rightarrow$ $\check{Y} \times \check{\mathscr{B}}$ is one-one and codes a separation of the first components of its range. Then some subset of $Y$ of cardinality $\lambda$ is separated.

Proof. Let $P=\bigcup_{\alpha<\kappa} P_{\alpha}$. Let $X_{\alpha}=\left\{\beta \in \lambda:\left(\exists p \in P_{\alpha}\right) p \| \dot{f}(\check{\beta})\right\}$. Then some $\left|X_{\alpha_{0}}\right|=\lambda$. For $\beta \in X_{\alpha_{0}}$, let $g(\beta)$ be that $\langle y, U\rangle$ such that for some $p \in P_{\alpha_{0}}$, $p \Vdash \dot{f}(\check{\beta})=\langle\check{y}, \check{U}\rangle$. Since $P_{\alpha_{0}}$ is centred, $g$ is well-defined and one-one and codes a separation of the first components of its range.

The general machinery used in the proof of Theorem 1(a), together with these two lemmas leads to a proof of $1(\mathrm{~b})$; however first we need another technical lemma.

Lemma 6. Suppose $\mathscr{Y}$ is a discrete collection in a topological space $X$. Suppose each point in $\bigcup \mathscr{Y}$ has character $\leq|\bigcup \mathscr{Y}|$. Then there is a subspace $X^{\prime}$ of $X$ of cardinality $|\bigcup \mathscr{Y}|$ including $\bigcup \mathscr{Y}$ such that for any $\mathscr{Z}=\left\{Z_{Y}\right\}_{Y \in \mathscr{Y}}, Z_{Y} \subseteq Y$, $\mathscr{Z}$ is separated in $X$ if and only if it is separated in $X^{\prime}$.

Proof. Fix a basis $\mathscr{B}_{y}$ for each $y \in \bigcup \mathscr{Y},\left|\mathscr{B}_{y}\right| \leq|\cup \mathscr{Y}|$. Pick a point in each nonempty intersection of elements of $\mathscr{B}_{y}$ and $\mathscr{B}_{y^{\prime}}$, for all pairs of distinct elements $y, y^{\prime}$ of $\bigcup \mathscr{Y}$. Let $X^{\prime}$ be $\bigcup \mathscr{Y}$ together with all these points.

Now, to prove (b), suppose $Y$ is a closed discrete set of size $\aleph_{2}$ in the first countable weakly $\aleph_{1}$-collectionwise Hausdorff space $\langle X, \mathscr{B}\rangle$. By Lemma 6, without loss of generality assume $|X|=|\mathscr{B}|=\aleph_{2}$. By first countability, as mentioned previously, $\tilde{X}=\left\langle j^{\prime \prime} X,\left\{j^{\prime \prime} B: B \in \mathscr{B}\right\}\right\rangle$ is a subspace of $\langle j(X), j(\mathscr{B})\rangle$; by generic hugeness, $j^{\prime \prime} X, j^{\prime \prime} \mathscr{B}, j^{\prime \prime} Y$ are all in $M\left[G_{j(\mathscr{P}) * j(\mathscr{R})}\right]$. Since $\langle j(X), j(\mathscr{B})\rangle$ is weakly $j\left(\aleph_{1}\right)$-collectionwise Hausdorff, some subset of $Y$ of cardinality $|Y|$ is separated in $\langle j(X), j(\mathscr{B})\rangle$ and hence in $\tilde{X}$. Separations are upward absolute, so the same is true in $V\left[G_{j(\mathscr{P}) * j(\mathscr{R})}\right] . \tilde{X}$ is homeomorphic to $\langle X, \mathscr{B}\rangle$ so in $V\left[G_{j(\mathscr{P}) * j(\mathscr{R})}\right]$, indeed some subset of $Y$ of cardinality $|Y|$ is separated in $\langle X, \mathscr{B}\rangle$. Now Lemmas 4 and 5 complete the proof.

(c) is proved by similar methods, plus a little bit of topology. The method of proof of Lemma 4 establishes

Lemma 7. If $\mathscr{Y}$ is a discrete collection with $|\bigcup \mathscr{Y}|=\lambda$ and $\mathscr{Y}$ can be separated via $\lambda$-closed forcing, then $\mathscr{Y}$ is separated.

Similarly, the method of proof of Lemma 5 establishes 
Lemma 8. If $\mathscr{Y}$ is a discrete collection in $V$ such that $\mathscr{Y}$ is separated in an extension via a $\kappa$-centered partial order, then there are in $V \kappa$ collections of disjoint open sets, $\left\{\mathscr{U}_{\alpha}\right\}_{\alpha<\kappa}$, such that $\bigcup \mathscr{Y} \subseteq \bigcup_{\alpha<\kappa} \cup \mathscr{U}_{\alpha}$.

By our general machinery and these lemmas, we will wind up with collections $\left\{\mathscr{U}_{\alpha}\right\}_{\alpha<\omega_{2}}$ of disjoint open sets, such that each element of $\bigcup \mathscr{Y}$ is in some member of one of them. Without loss of generality, since in this case we are dealing with points, we may then assume each open set in question satisfies the $\aleph_{2}$-c.c. But then $\bigcup_{\alpha<\omega_{2}} \mathscr{U}_{\alpha}$ is star- $\aleph_{2}$, i.e. each element of it meets fewer than $\aleph_{2}$ other elements of it. We may also assume, by the discreteness of $\mathscr{Y}$, that each element of $\bigcup_{\alpha<\omega_{2}} \mathscr{U}_{\alpha}$ meets at most one element of $\mathscr{Y}$. The following is well-known $\left[\mathrm{T}_{1}\right]$ and completes the proof of $(\mathrm{c})$ :

Lemma 9. Suppose $\mathscr{Y}$ is disjoint and every subcollection of $\mathscr{Y}$ of power $<\kappa$, $\kappa$ regular, is separated. If there is a star- $\kappa$ collection of open sets covering $\bigcup \mathscr{Y}$, each meeting at most one element of $\mathscr{Y}$, then $\mathscr{Y}$ is separated.

(d), (e), (f), and (g) are variations on a theme. (e) follows from (d) since $Y \times$ $\left(\omega_{1}+1\right)$ normal $\left(\omega_{1}+1\right.$ is given the order topology) implies $Y$ is $\aleph_{1}$-paracompact (see e.g. [P]), while the normality, respectively $\aleph_{1}$-paracompactness, of open subspaces easily implies hereditary properties. (g) follows from (f) since $\aleph_{1}$-collectionwise normal Moore spaces are $\aleph_{1}$-paracompact, and collectionwise normal Moore spaces are metrizable. We will prove $(\mathrm{d})$ - the main result-in the Hausdorff case first, and then observe that by detouring to prove (f) (via a similar proof), we can avoid the Hausdorff assumption.

Our first attempt at a proof of (d) was straightforward: use the hereditary $j\left(\aleph_{1}\right)$-paracompactness of $j(X)$ to obtain a locally finite refinement of $\left\{j^{\prime \prime} U: U \in \mathscr{U}\right\} \mathscr{U}$ an open cover of $X$. Pull it back in usual fashion to obtain $\aleph_{1}$ open collections $\left\{\mathscr{U}_{\alpha}\right\}_{\alpha<\omega_{1}}$ refining $\mathscr{U}$ such that $\bigcup_{\alpha<\omega_{2}} \mathscr{U}_{\alpha}$ covers $X$. Use the centredness to argue that $\mathscr{U}_{\alpha}$ is locally finite on $\bigcup \mathscr{U}_{\alpha}$ and then use the $\aleph_{1}$-paracompactness of $X$ in standard topological fashion to transform our $\aleph_{1}$ refinements into a single locally finite refinement of $\mathscr{U}$. There is a serious technical difficulty however; it is not at all evident that the locally finite refinement of $\left\{j^{\prime \prime} U: U \in \mathscr{U}\right\}$ can be assumed to be composed of $j^{\prime \prime}$-images of open subsets of $X$. This would be needed to "represent" the refinement in the ground model. We therefore proceed via a slightly circuitous route which requires us to assume normality. Normality will enable us to get away with transferring down disjointness instead of local finiteness. The next two lemmas indeed say that disjointness goes down.

Lemma 10. Let $P=\bigcup_{\gamma<\kappa} P_{\gamma}$ be $\kappa$-centred. Let $\mathscr{S}$ be an open cover of $X$. Let $\mathscr{U}$ be a family of $\kappa$ disjoint open collections such that $1 \Vdash \dot{\mathscr{U}}$ refines $\check{\mathscr{S}}$. Then $\exists \mathscr{W} \in V$ refining $\mathscr{S}, \mathscr{W}$ also a family of $\kappa$ disjoint open collections.

Proof. Consider $\mathscr{U}$ mapping $\kappa \times \eta$ into $V^{\mathscr{P}}$, such that for fixed $\beta \in \kappa$, $\mathscr{U} \mid(\{\beta\} \times \eta)$ lists a disjoint open collection (possibly repeatedly listing empty sets). For $\beta, \gamma<\kappa$ and $\alpha<\eta$, let

$$
W_{\beta \gamma \alpha}=\bigcup\left\{W \in \mathscr{T}:\left(\exists p \in P_{\gamma}\right)(\exists S \in \mathscr{S})(p \Vdash \check{W} \subseteq \dot{\mathscr{U}}(\beta, \alpha) \subseteq \check{S})\right\} .
$$

Then $\mathscr{W}=\left\{W_{\beta \gamma \alpha}: \beta, \gamma<\kappa, \alpha<\eta\right\}$ covers, since $\mathscr{U}$ covers and refines $\mathscr{S}$. $\mathscr{W}$ refines $\mathscr{S}$; to see this, take $x \in W_{\beta \gamma \alpha}$. Then $x \in$ some $W$ such that 
for some $p \in P_{\gamma}$ there is an $S$ such that $p \Vdash \check{W} \subseteq \check{S}$, so $W \subseteq S$. Claim $W_{\beta \gamma \alpha} \subseteq S$. For given any $W^{\prime}$ and a $p^{\prime} \in P_{\gamma}$ such that $p^{\prime} \Vdash \check{W}^{\prime} \subseteq \dot{\mathscr{U}}(\beta, \alpha)$, take $q \leq p, p^{\prime}$. Then $q \Vdash \check{W}^{\prime} \subseteq \check{S}$.

Finally, observe that for $\alpha \neq \alpha^{\prime}, W_{\beta \gamma \alpha} \cap W_{\beta \gamma \alpha^{\prime}}=0$ since $P_{\gamma}$ is centred and $\mathscr{U} \mid(\{\boldsymbol{\beta}\} \times \eta)$ is disjoint.

Next we prove

Lemma 11. If $|X|=\lambda, \mathscr{P}$ is $\lambda$-closed, $\mathscr{S}$ is an open cover and $1 \Vdash$ " $\check{\mathscr{S}}$ has a $\kappa$-disjoint refinement", then it has one in $V$.

Proof. Let $\mathscr{U}: \kappa \times \lambda \rightarrow V^{\mathscr{P}}$ as in the previous lemma. For every $x \in X$,

$$
\begin{aligned}
D_{x}=\{p:(\exists \beta<\kappa)(\exists \alpha<\lambda)(\exists S \in \mathscr{S})(\exists W \in \mathscr{T}) & \\
& (x \in W \& p \Vdash \check{W} \subseteq \dot{\mathscr{U}}(\beta, \alpha) \subseteq \check{S})\}
\end{aligned}
$$

is dense. Let $X=\left\{x_{\gamma}\right\}_{\gamma<\lambda}$ and pick a descending sequence of conditions $\left\{p_{\gamma}\right\}_{\gamma<\lambda}, P_{\gamma} \in D_{x_{\gamma}}$. Pick $\beta_{\gamma}, \alpha_{\gamma}, W_{\gamma}$ such that $x_{\gamma} \in W_{\gamma} \in \mathscr{T}$ and $(\exists S \in$ $\mathscr{S})\left(p_{\gamma} \Vdash \check{W}_{\gamma} \subseteq \dot{\mathscr{U}}\left(\beta_{\gamma}, \alpha_{\gamma}\right) \subseteq \check{S}\right)$. Let

$$
W_{\beta \alpha}=\bigcup\left\{W_{\gamma}: p_{\gamma} \Vdash \check{W}_{\gamma} \subseteq \dot{\mathscr{U}}(\beta, \alpha)\right\} .
$$

$\left\{W_{\beta \alpha}: \beta<\kappa, \alpha<\lambda\right\}$ certainly covers. The same argument as in the proof of the previous lemma shows it refines $\mathscr{S}$ and is $\kappa$-disjoint.

We now need to do some reasonably standard work with covering properties so as to be able to obtain the required collectionwise normality and paracompactness from the kind of refinements obtainable via Lemmas 10 and 11. Bibliographic references and standard proofs can be found e.g. in [E]. We will give somewhat more details than we might otherwise, for the benefit of set theorists trying to follow the topological arguments. Alternatively they could skip to the end of Lemma 19, after which the elementary embedding argument is given that completes the proof.

In dealing with covers and refinements, we tacitly assume them to be open unless stated otherwise. The following well-known definition and lemma are very convenient.

Definition. A refinement $\left\{V_{\alpha}\right\}_{\alpha \in B}$ of the cover $\left\{U_{\alpha}\right\}_{\alpha \in A}$ is said to be precise if $B=A$ and, for each $\alpha, V_{\alpha} \subseteq U_{\alpha}$.

Lemma 12. If a cover has a point-finite (locally finite) refinement, it has a precise one.

Proof. Routine.

Lemma 13. If $X$ is $\kappa$-paracompact ( $\kappa$-metacompact) and $\mathscr{U}$ is an open cover of $X$ such that $\mathscr{U}=\bigcup_{\alpha<\kappa} \mathscr{U}_{\alpha}$, where each $\mathscr{U}_{\alpha}$ is locally (point-) finite, then $\mathscr{U}$ has a locally (point-) finite refinement.

Proof. Let $U_{\alpha}=\bigcup \mathscr{U}_{\alpha}$. Let $\left\{V_{\alpha}\right\}_{\alpha<\kappa}$ be a locally (respectively, point-) finite precise refinement of $\left\{U_{\alpha}\right\}_{\alpha<\kappa}$. Then $\left\{V_{\alpha} \cap U: U \in \mathscr{U}_{\alpha}, \alpha<\kappa\right\}$ is a locally (respectively, point-) finite refinement.

Lemma 14. Normal countably metacompact spaces are countably paracompact.

This is standard-see e.g. [E, 5.2.6]. 
Lemma 15. If a discrete collection is separated in a normal space, then it has a discrete separation.

Proof. This is a standard exercise-see e.g. [E, 5.1.17].

We twice need the proof-not just the (standard) result that metacompact collectionwise normal spaces are paracompact in what follows. For the benefit of the reader, we give it here, in a slightly sharper version.

Lemma 16. If $X$ is $\lambda$-metacompact and $\lambda$-collectionwise normal, it is $\lambda$-paracompact, and in fact, every open cover of size $\leq \lambda$ has a $\sigma$-discrete refinement (i.e. a refinement which is the union of countably many discrete collections of open sets).

Proof. Without loss of generality, assume $\mathscr{U}=\left\{U_{\alpha}\right\}_{\alpha<\lambda}$ is a point-finite open cover. We will produce a $\sigma$-discrete refinement $\left\{V_{n}\right\}_{n<\omega}$ of $\mathscr{U}$ of cardinality $\lambda$. By Lemmas 13 and 14 it will follow in addition that $\mathscr{U}$ has a locally finite refinement. We define $\mathscr{V}_{0}=\{\varnothing\}$ and for $n>0$ we shall recursively define $\mathscr{V}_{n}=\left\{V_{T}: T \in[\lambda]^{n}\right\}$ to be a discrete family of open sets, each of which is included in a member of $\mathscr{U}$, and such that, letting $W_{n}=\bigcup \mathscr{\mathscr { V }}_{n}$, if $\left|\left\{\alpha<\lambda: x \in U_{\alpha}\right\}\right| \leq n$, then $x \in \bigcup_{i=0}^{n} W_{i}$. Given the $\mathscr{V}_{n}$ 's for $n \leq m$, for each $T \in[\lambda]^{m+1}$ let $A_{T}=\left(X-\bigcup_{i=0}^{n} W_{n}\right) \cap\left(X-\bigcup_{\alpha \notin T} U_{\alpha}\right)$. Then $A_{T} \subseteq \bigcap_{\alpha \in T} U_{\alpha}$. Claim $\left\{A_{T}\right\}_{T \in[\lambda]^{m+1}}$ is discrete. This is done by cases. Let $F=\left\{\alpha: x \in U_{\alpha}\right\}$. If $|F|=m+2$, then $\bigcap\left\{U_{\alpha}: \alpha \in F\right\}$ meets no $A_{T}, T \in[\lambda]^{m+1}$. If $|F| \leq m$, $\bigcup_{i=0}^{n} W_{m}$ is a neighbourhood of $x$ disjoint from those $A_{T}$ 's. If $|F|=m+1$, $\bigcap\left\{U_{\alpha}: \alpha \in F\right\}$ meets $A_{F}$ and no other $A_{T}, T \in[\lambda]^{m+1}$.

By Lemma 15 , let $\left\{S_{T}: T \in[\lambda]^{m+1}\right\}$ be a discrete family of open sets separating $\left\{A_{T}: T \in[\lambda]^{m+1}\right\}$. Let $V_{T}=S_{T} \cap \bigcup_{\alpha \in T} U_{\alpha}$. Then $\mathscr{V}_{m}=\left\{V_{T}: T \in[\lambda]^{m+1}\right\}$ is as required. Since $\mathscr{U}$ is point-finite, $\mathscr{V}=\bigcup_{m<\omega} \mathscr{V}_{m}$ is the required discrete refinement of cardinality $\lambda^{<\omega}=\lambda$.

Lemma 17. If $\mathscr{U}=\left\{U_{\alpha}\right\}_{\alpha<\lambda}$ is a point-finite open cover of a normal space, there exists an open cover $\mathscr{V}=\left\{V_{\alpha}\right\}_{\alpha<\lambda}$ such that for every $\alpha, \bar{V}_{\alpha} \subseteq U_{\alpha}$.

This is yet another standard result about coverings-see e.g. [E.1.5.18]. Also standard is that paracompact normal spaces are collectionwise normal (the technique of proof can be found embedded in the proof of Lemma 19); (hence) so are paracompact Hausdorff spaces. We shall need

Lemma 18. $\lambda$-paracompact normal spaces are $\lambda$-collectionwise normal.

The proof is standard.

Lemma 19. For any $\kappa$ and $\lambda$, normal $\kappa$-paracompact $\lambda$ - $\kappa$-screenable spaces are $\lambda$-paracompact.

This is the topological key-we will get paracompactness once we have transferred down disjointness.

Proof. Obviously we may assume $\kappa \leq \lambda$. By Lemma 16, it suffices to prove such spaces are $\lambda$-metacompact and $\lambda$-collectionwise normal. $\lambda$-metacompactness is immediate via Lemma 13. To obtain $\lambda$-collectionwise normality, given a discrete collection $\mathscr{Y}=\left\{Y_{\gamma}\right\}_{\gamma<\lambda^{\prime}}, \lambda^{\prime} \leq \lambda$, claim it will suffice to find discrete open collections $\left\{\mathscr{V}_{\alpha}\right\}_{\alpha<\kappa}$ such that $\bigcup \mathscr{Y} \subseteq \bigcup\left\{\mathscr{V}_{\alpha}: \alpha<\kappa\right\}$ and each $V$ in each 
$\mathscr{V}_{\alpha}$ meets at most one element of $\mathscr{Y}$. For then let $\left\{W_{\alpha}\right\}_{\alpha<\kappa}$ be a precise locally finite refinement of $\{X-\overline{\cup \mathscr{Y}}\} \cup\left\{\cup \mathscr{V}_{\alpha}\right\}_{\alpha<\kappa}$. Then $\mathscr{U}=\left\{V \cap W_{\alpha}: V \in \mathscr{V}_{\alpha}: \alpha<\right.$ $\kappa\}$ is locally finite and so

$$
\left\{\bigcup\left\{U \in \mathscr{U}: U \in Y_{\gamma} \neq 0\right\}-\overline{\bigcup\left\{U \in \mathscr{U}: U \cap Y_{\gamma}=0\right\}}: \gamma<\lambda^{\prime}\right\}
$$

is the required separation. By hypothesis we have open collections as called for in the claim except that they are disjoint rather than discrete, so we shall shrink them to make them discrete. First observe that we could have without loss of generality assumed $\mathscr{Y}$ is a discrete collection of closed sets and hence that $\bigcup \mathscr{Y}$ is closed. It follows that $\bigcup \mathscr{Y}$ is normal and $\lambda$-metacompact. By Lemma 17, then, there exist $T_{\alpha}$ closed in $\bigcup \mathscr{Y}$ (and hence closed) such that $T_{\alpha} \subseteq \bigcup \mathscr{V}_{\alpha} \cap \bigcup \mathscr{Y}$. For each $\alpha,\left\{Y_{\gamma} \cap T_{\alpha}: \gamma<\lambda^{\prime}\right\}$ is a discrete collection separated by the open sets $V_{\alpha, \gamma}=\bigcup\left\{V \in \mathscr{V}_{\alpha}: V \cap Y_{\gamma} \neq 0\right\}$. By normality, by Lemma 15 we can then find for each $\alpha$ a discrete collection of open sets $\left\{V_{\alpha, \gamma}^{\prime}\right\}_{\gamma<\lambda^{\prime}}, Y_{\gamma} \cap T_{\alpha} \subseteq V_{\alpha, \gamma}^{\prime} \subseteq V_{\alpha, \gamma}$ which is what we wanted.

We could prove (d) now if we were prepared to assume $X$ Hausdorff. (In fact I have set the proof up in the way I did so that I could get away without that assumption.) To see this, in $M\left[G_{j(\mathscr{P}) * j(\mathscr{R})}\right]$ we have $\widetilde{X}=\left\langle j^{\prime \prime} X,\left\{j^{\prime \prime} B: B \in\right.\right.$ $\mathscr{B}\}\rangle$ is a subspace of the hereditarily $j\left(\aleph_{1}\right)$-paracompact space $\langle j(X), j(\mathscr{B})\rangle$. Therefore $\widetilde{X}$ is $j\left(\aleph_{1}\right)$-paracompact and hence paracompact. It is also paracompact in $V\left[G_{j(\mathscr{P}) * j(\dot{\mathscr{R}})}\right]$ since any open cover has a refinement consisting of $j\left(\aleph_{1}\right)$-many $j^{\prime \prime} B^{\prime}$ 's, and such refinements lie in $M\left[G_{j(\mathscr{P}) * j(\mathscr{R})}\right]$. It is Hausdorff there if it is in $V\left[G_{\mathscr{P}_{*} \dot{\mathscr{R}}}\right]$ since Hausdorffness is preserved by any forcing. As mentioned before Lemma 18, paracompact Hausdorff spaces are collectionwise normal, so by Lemma $16, \widetilde{X}$ and hence its homeomorphic copy $X$ is screenable in $V\left[G_{j(\mathscr{P}) * j(\dot{\mathscr{R}})}\right]$. By Lemmas 10 and $11, X$ is $\aleph_{2}-\aleph_{1}$-screenable in $V\left[G_{\mathscr{P} * \dot{\mathscr{R}}}\right]$, after which Lemma 19 finishes the proof.

Our only use of Hausdorffness in the above was to obtain collectionwise normality, but we can get that without using the Hausdorff assumption. The general machinery should by now be familiar-by Lemma 6 , assume $|X|=\aleph_{2}$ and again by first countability, let $\mathscr{B}$ be a base of cardinality $\leq \aleph_{2} . j(\mathscr{Y})$ is discrete in $\langle j(X), j(\mathscr{B})\rangle$ and hence so is $\widetilde{\mathscr{Y}}=\left\{j^{\prime \prime} Y: Y \in \mathscr{Y}\right\}$, which is indeed in $M\left[G_{j(\mathscr{P}) * j(\mathscr{R})}\right]$ as is $\widetilde{X}$ defined as before. Since $\langle j(X), j(\mathscr{B})\rangle$ is normal and $j\left(\aleph_{1}\right)$-paracompact, $\widetilde{\mathscr{Y}}$ is separated there. The usual argument provides for $\langle X, \mathscr{B}\rangle$ in $V\left[G_{\mathscr{D}_{*} \mathscr{R}}\right] \aleph_{1}$ disjoint open collections whose union covers $\mathscr{Y}$ and such that each member of each collection meets at most one element of $\mathscr{Y}$. But now we can proceed to separate $\mathscr{Y}$ using the proof of $\lambda$-collectionwise normality in the proof of Lemma 19.

\section{EXAMPLES}

We have counterexamples for most parts of Theorem 1, showing that more than ZFC or indeed ZFC + GCH is needed. At the end of the paper we will explicitly state the more interesting open problems. Here are the examples:

Example 1. After I raised the question in the first draft of this paper, L. Soukup [So] forced to obtain a model of GCH containing a nonspecial Aronszajn $\omega_{2}$ tree $T$ such that each $T \mid \alpha$ is special for all $\alpha<\omega_{2}$. Todorčević obtained 
a better result [To]: if $\omega_{2}$ is not weakly compact in $L$, there is a nonspecial Aronszajn $\omega_{2}$-tree $T$ such that $T \mid \alpha$ is special for all $\alpha<\omega_{2}$.

Example 2. I am grateful to Mary Ellen Rudin for informing me of the following Fact. Let $\lambda$ and $\kappa$ be infinite cardinals. Let $X$ be a subspace of $\kappa, \kappa$ given the order topology. Then $X$ is $\lambda$-paracompact if and only if for all $\alpha \in(\kappa+1)-X$ with $\aleph_{1} \leq \operatorname{cf}(\alpha) \leq \lambda, X \cap \alpha$ is not stationary in $\alpha$.

I leave the proof to the reader.

The axiom $E\left(\omega_{2}\right)$ asserts the existence of a stationary set $E \subseteq\left\{\alpha \in \omega_{2}: \operatorname{cf}(\alpha)\right.$ $=\omega\}$ such that for no limit $\delta<\omega_{2}$ is $E \cap \delta$ stationary in $\delta . E\left(\omega_{2}\right)$ follows from $V=L$ and so is consistent with $\mathrm{GCH}$. As a subspace of $\omega_{2}$ given the order topology, $E$ is a first countable hereditarily collectionwise normal space of cardinality $\aleph_{2}$. It follows immediately from the Fact that $E$ is (hereditarily) $\aleph_{1}$-paracompact but not paracompact. Thus (d) of Theorem 1 does not follow from GCH.

For further results concerning stationary sets and paracompactness, see Theorem 25 and Theorem 30 et seq below.

Example 3. $E\left(\omega_{2}\right)$ can also be used to obtain counterexamples to other portions of Theorem 1. For each $\alpha \in E$, choose $s_{\alpha}: \omega \rightarrow \alpha$ to be a strictly increasing sequence converging to $\alpha$. Let $D=\left\{s_{\alpha} \mid m: \alpha \in E, m \in \omega\right\}$. Let $X=E \cup D$. The points of $D$ are taken to be isolated; the $n$th neighbourhood of $\alpha \in E$ is $N(\alpha, n)=\{\alpha\} \cup\left\{s_{\alpha} \mid m: m>n\right\}$. This space is due to Fleissner [F $\left.\mathrm{F}_{3}\right]$. See also $\left[\mathrm{S}_{1}\right]$. It is easy to see $\left[\mathrm{F}_{3}\right]$ that $X$ is a locally compact, locally countable Moore space which is $\aleph_{1}$-collectionwise Hausdorff but not $\aleph_{2}$-collectionwise Hausdorff. Thus (c) of Theorem 1 does not follow from $\mathrm{GCH}$. In fact, since $E$ is closed discrete and $D$ is isolated, $\aleph_{1}$-collectionwise Hausdorff implies $\aleph_{1}-\aleph_{1}$ collectionwise normal. Also observe that the space is weakly $\aleph_{2}$-collectionwise Hausdorff, for just inductively pick $\alpha$ 's and $n_{\alpha}$ 's such that $N\left(\alpha, n_{\alpha}\right)$ is disjoint from all the $N\left(\beta, n_{\beta}\right)$ chosen previously. Since neighbourhoods "look back", this can clearly be done. Since $X$ is first countable but not collectionwise Hausdorff, by $\left[\mathrm{F}_{1}\right] V=L$ implies $X$ is not normal. In $\left[\mathrm{S}_{1}\right]$ it is shown that Martin's Axiom plus $2^{\aleph_{0}}>\aleph_{2}$ implies $X$ is normal, and that there is a forcing extension in which $2^{\aleph_{0}}=\aleph_{1}$ and $2^{\aleph_{1}}=\aleph_{3}$ in which $X$ is normal. GCH implies $X$ is not $\aleph_{1}$-collectionwise normal, since Fleissner $\left[\mathrm{F}_{5}\right]$ observed that it implies first countable $\aleph_{1}$-collectionwise normal spaces are $\aleph_{2}$-collectionwise Hausdorff. One can probably combine the methods of $\left[S_{1}\right.$ and $\left.S K\right]$ to construct a particular $X$ which can be forced to be $\aleph_{1}$-collectionwise normal, keeping $\mathrm{CH}$, but I have not checked the details. It is not known whether GCH implies normal first countable $\aleph_{1}$-collectionwise Hausdorff spaces are $\aleph_{2}$-collectionwise Hausdorff; it does not imply the result for spaces of character $\leq \aleph_{1}$. The reason is that in [SK] a model of GCH is constructed in which for each $\delta \in S=\{\delta \in$ $\left.\omega_{2}: \operatorname{cf}(\delta)=\omega_{1}\right\}$, there is an increasing $\omega_{1}$-sequence $\eta_{\delta} \rightarrow \delta$, such that for each $c=\left\langle c_{\delta}: \delta \in S\right.$ and $\left.c_{\delta} \in \omega_{1} 2\right\rangle$ there is an $f: \omega_{2} \rightarrow 2$ so that for each $\delta \in S$ there is $\mu_{\delta}<\omega_{1}$ such that for all $\mu>\mu_{\delta}, \mu \in \omega_{1}, f\left(\eta_{\delta}(\mu)\right)=c_{\delta}(\mu)$. (For those who know the terminology, we say $S$ has the uniformization property for $\eta=\left\langle\eta_{\delta}: \delta \in S\right\rangle$.) If one constructs the analog of a ladder system space for $\eta$, one obtains the required counterexample. The point is that since $S$ is stationary, the space is not $\aleph_{2}$-collectionwise Hausdorff, uniformization yields normality, and normality plus $G_{\delta}$ 's open yields $\aleph_{1}$-collectionwise normality. 
Example 4. I do not have a consistent-with-GCH example of a first countable weakly $\aleph_{1}$-collectionwise Hausdorff space which is not weakly $\aleph_{2}$-collectionwise Hausdorff; there is however a consistent-with-GCH example of an $\aleph_{1}$-collectionwise Hausdorff space of character $\aleph_{1}$ which is not weakly $\aleph_{2}$-collectionwise Hausdorff. I describe it in $\left[\mathrm{T}_{7}\right]$; it is just the usual tangent disk space "lifted" one cardinal: take the completion of an $\eta_{1}$-set, isolate the $\eta_{1}$-set, and for each of the remaining points pick an $\omega_{1}$-sequence from the $\eta_{1}$-set converging to it, letting neighbourhoods be tails. Assuming $\mathrm{CH}$, the $\eta_{1}$-set has cardinality $\aleph_{1}$ while its completion has cardinality $2^{\aleph_{1}}$. It follows that the space cannot be weakly $\aleph_{2}$-collectionwise Hausdorff since the $\eta_{1}$-set is dense while its remainder is closed discrete. On the other hand, the space is $\aleph_{1}$-collectionwise Hausdorff since it is regular and $G_{\delta}$ 's are open. Again under $\mathrm{CH}$, the space has local cardinality $\aleph_{1}$, showing that first countability is necessary in the collectionwise Hausdorff case of $1(\mathrm{c})$. Assuming the axiom BACH plus $2^{\aleph_{1}}>\aleph_{2}$, a subspace of the space can be made normal (and hence $\aleph_{1}$-collectionwise normal), while retaining the other properties $\left[T_{7}\right]$.

Example 5. P. Daniels [D], in response to the first draft of this paper, constructed from Martin's Axiom plus $2^{\aleph_{0}}=\aleph_{2}$ a first countable $\aleph_{1}$-collectionwise Hausdorff space which is not weakly $\aleph_{2}$-collectionwise Hausdorff.

Example 6. $\mathfrak{p}=\mathfrak{c}$ asserts that if $\left\{A_{\alpha}\right\}_{\alpha<\lambda}, \lambda<2^{\aleph_{0}}$, are infinite subsets of $\omega$ with each finite intersection of the $A_{\alpha}$ 's infinite, there is an infinite $A \subseteq \omega$ such that $A-A_{\alpha}$ is finite for all $\alpha . \mathfrak{p}=\mathfrak{c}$ is equivalent [B] to Martin's Axiom for $\aleph_{0}$-centred partial orders and implies there is a first countable $<2^{\aleph_{0}}$ collectionwise Hausdorff space which is not $2^{\aleph_{0}}$-collectionwise Hausdorff [vD] (also see $\left[D_{T W}\right.$ and $\left.F_{6}\right]$ ). If $\mathfrak{p}=\mathfrak{c}$ is forced in the usual way, with ground model $L$, by standard techniques (see e.g. $\left[\mathrm{T}_{4}\right]$ ) one gets that normal spaces of character $\leq 2^{\aleph_{0}}$ which are $<2^{\aleph_{0}}$-collectionwise Hausdorff are collectionwise Hausdorff. In an earlier version of this paper I used a different argument, involving a supercompact cardinal, to obtain this reflection. I am grateful to Bill Fleissner for suggesting that I did not need a large cardinal. Van Douwen's example is in fact $<2^{\aleph_{0}}-<2^{\aleph_{0}}$-collectionwise normal; using the supercompact, I can get a model for $\mathfrak{p}=\mathfrak{c}$ in which normal countably metacompact spaces with $\hat{\chi} \leq 2^{\aleph_{0}}$ are collectionwise normal if they are $<2^{\aleph_{0}}-<2^{\aleph_{0}}$ collectionwise normal $\left[\mathrm{T}_{8}\right]$.

Example 7. Sometimes we can transfer from $\aleph_{1}$ to get a structure of size $\aleph_{2}$ being the union of $\aleph_{1}$ nice substructures, and yet be provably unable to fully transfer to $\aleph_{2}$. In [MS], Milner and Shelah give an example (due to Truss) of a family $\mathscr{F}$ of $\aleph_{2}$ countable sets such that every subfamily of size $\leq \aleph_{1}$ has a transversal (i.e. a one-one choice function) but the whole family does not. The usual proof, however, yields in the Foreman-Laver model $\aleph_{1}$ subfamilies of $\mathscr{F}$, whose union is the whole family, such that each has a transversal.

\section{BEYOND $\aleph_{2}$}

The " $\aleph_{1}-\aleph_{2}$ " results proved so far cry out for generalization. For a fixed regular $\kappa$, one can replace " $\aleph_{1}-\aleph_{2}$ " by " $\kappa^{+}-\kappa^{++}$" by a straightforward variation of the Foreman-Laver model. It is more interesting however to attempt the transfer for all regular $\kappa$ simultaneously, or even for all $\kappa$. Foreman did 
something of this sort in $\left[\mathrm{Fo}_{2}\right]$, where from a huge cardinal he obtained $\kappa^{+}$saturated ideals on all regular $\kappa$, but it is not yet clear how far this can be pushed. It is therefore at this stage more instructive to postulate the existence of appropriate "generic huge embeddings" and see what consequences we can derive, deferring the difficult task of showing the consistency of such existence. (They do not obviously exist in $\left[\mathrm{Fo}_{2}\right]$.) This follows the program put forward in $\left[\mathrm{Fo}_{3}\right]$.

Definition [ $\left[\mathrm{Fo}_{3}\right]$. If $j: V \rightarrow M$ is an elementary embedding, then $j$ is called generic if there is a partial order $\mathscr{P}$ such that $j$ is definable in $V\left[G_{\mathscr{P}}\right]$ (thus $M$ is a transitive subclass of $\left.V\left(G_{\mathscr{P}}\right]\right)$.

The reader should think in particular of the extended elementary embedding $j$ mentioned in clause ii) of our description of the Foreman-Laver model. This extended $j$ is generic over $V\left[G_{\mathscr{P}_{*} \dot{\mathscr{R}}}\right]$. As Foreman points out, there are three important parameters to be used in classifying generic embeddings: the degree of closure of $M$, where ordinals are moved, and the nature of the forcing.

Definition. Let $j: V \rightarrow M$ be a generic elementary embedding. Let $\kappa_{0}$ be the critical point of $j$ and, for $i \in \omega, \kappa_{i+1}=j\left(\kappa_{i}\right) . j$ is generically $n$-huge if $M$ is closed under $\kappa_{n}$-sequences. If $n=1$, we simply say "huge".

In $\left[\mathrm{Fo}_{3}\right]$, Foreman uses the weaker condition that $\mathscr{P}\left(\kappa_{n}\right) \subseteq M$. Foreman is concerned with generic embeddings moving finite increasing sequences of regular cardinals to other increasing sequences of the same length. For the sorts of applications we are interested in, the main cases of interest are (a) moving $\aleph_{1}$ somewhere, and (b) moving "many" cardinals to their successors. As for the forcing, Foreman examines its saturation (i.e. chain condition) properties. These of course are crucial, but we want to also (following a suggestion of Foreman) consider decomposing $\mathscr{P}$ into a two-step iteration, making use-as in the Foreman-Laver model-of both chain condition and closure properties.

Foreman derives the existence of some generic elementary embeddings from the existence of highly saturated "higher order" ideals. Since for the more interesting embeddings, these ideals are not known to be consistent, even relative to large cardinals, there is no point in pursuing that topic here. For our purposes here it is neater to take the existence of the embeddings as axiomatic, rather than to postulate the ideals, but, as the referee notes, the advantage of taking the existence of the ideals as axioms is that this is a first-order extension of ZFC.

The Foreman-Laver model establishes the consistency, relative to a huge cardinal, of a generic huge embedding which sends $\aleph_{1}$ to $\aleph_{2}$ and is such that the forcing is $\aleph_{1}$-centred followed by $\aleph_{2}$-closed. The existence of such an embedding is all we have used about huge cardinals so far.

We can eliminate the possibility of moving $\aleph_{1}$ to a cardinal greater than $2^{\aleph_{1}}$ if we wish to retain all useful properties. The existence of a generic huge embedding moving $\aleph_{1}$ to $\aleph_{n}>2^{\aleph_{1}}$, such that the forcing is $\aleph_{1}$-centred followed by $\aleph_{n}$-closed, implies the product of $\aleph_{n}$ copies of the two-point discrete space has a dense set of cardinality $\aleph_{1}$, which it does not.

To see this implication, consider the partial order $\mathscr{Q}$ of inclusion on the nonempty basic open sets of $\{0,1\}^{\aleph_{n}}$. It suffices to show that such an embedding would make $\mathscr{Q} \aleph_{1}$-centred. Note that $|Q|=\aleph_{n}$ and that each subset of 
$Q$ of size $\aleph_{1}$ is $\aleph_{0}$-centred. To see this latter fact, observe the set is supported on a product of $\aleph_{1}$ factors, which is separable. Now since $V\left[G_{\left(\mathscr{P}_{*} \mathscr{R}\right)}\right] \vDash$ " $\mathscr{Q}$ is a partial order of size $\aleph_{n}$ such that every subset of power $\aleph_{1}$ is $\aleph_{0}$-centred", then $M\left[G_{j(\mathscr{P}) * j(\dot{R})}\right] \vDash$ " $j(\mathscr{Q})$ is a partial order such that every subset of power $j\left(\aleph_{1}\right)$ is $\aleph_{0}$-centred". Since $j\left(\aleph_{1}\right)=|Q|$ and $M\left[G_{j(\mathscr{P}) * j(\mathscr{R})}\right]$ is closed under $j\left(\aleph_{1}\right)$-sequences, $M\left[G_{j(\mathscr{P}) * j(\dot{\mathscr{R}})}\right] \vDash$ “ $j(\mathscr{Q})$ is $\aleph_{0}$-centred". $\aleph_{0}$-centering is upward absolute so $V\left[G_{j(\mathscr{P}) * j(\dot{\mathscr{R}})}\right] \vDash$ “ $j(\mathscr{Q})$ is $\aleph_{0}$-centred". $\mathscr{Q}$ is isomorphic to $j^{\prime \prime} \mathscr{Q}$ so $V\left[G_{j(\mathscr{P}) * j(\dot{R})}\right] \vDash$ " $\mathscr{Q}$ is $\aleph_{0}$-centred".

Just as with the trees earlier, we can pull the centering back to $V\left[G_{\mathscr{P}_{*} \dot{\mathscr{R}}}\right]$ in two stages, obtaining the contradiction that $\mathscr{Q}$ is $\aleph_{1}$-centred. We state the required lemmas, but leave the proofs to the reader.

Lemma 20. If $\mathscr{S}$ is a $\lambda$-closed partial order and $|Q| \leq \lambda$ and $1 \Vdash \mathscr{S}$ “ $\check{\mathscr{S}}$ is $\check{\delta}$-centred", then $\mathscr{Q}$ is $\delta$-centred.

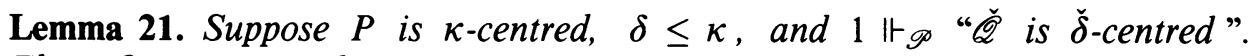
Then $\mathscr{Q}$ is $\kappa$-centred.

Given that we cannot have everything we want, there are several possibilites. One is to weaken " $\aleph_{1}$-centred" to " $\aleph_{2}$-chain condition". On course, since our proofs used centering heavily, it is not clear that this is of any use. But in fact slightly stronger properties are:

Definition. A partial order $\mathscr{P}$ has property $K_{\lambda}$ if every subset of size $\lambda$ includes a linked (i.e. pairwise compatible) subset of size $\lambda . \mathscr{P}$ has precaliber $\lambda$ if every subset of size $\lambda$ includes a centred subset of size $\lambda$.

Lemma 22. Suppose $\mathscr{P}$ has property $K_{\lambda}$ and $1 \Vdash_{\mathscr{P}}$ “ $\check{X}$ is weakly $\check{\lambda}$-collectionwise Hausdorff". Then $X$ is weakly $\lambda$-collectionwise Hausdorff.

Proof. This is standard by the technique of [KT]. Use property $K_{\lambda}$ to get $\lambda$ compatible conditions deciding the separation. Note this shows we could have used property $K_{\aleph_{2}}$ rather than $\aleph_{1}$-centred when we dealt with weak collectionwise Hausdorffness earlier in the paper.

We therefore have that if there is a generic huge embedding sending $\aleph_{1}$ to $\aleph_{n}$ definable by $K_{\aleph_{n}} * \aleph_{n}$-closed forcing, then first countable weakly $\aleph_{1}$ collectionwise Hausdorff spaces are weakly $\aleph_{n}$-collectionwise Hausdorff. As noted by the authors, one can modify the Foreman-Laver construction to collapse $j(\kappa)$ to $\aleph_{n}$ instead of $\aleph_{2}$. In this case, $j(\mathscr{P}) /(\mathscr{P} * \mathscr{R})$ will have property $K_{\aleph_{n}}$ (in fact it is $\aleph_{n-1}$-centred) while $j(\mathscr{R})$ is $\aleph_{n}$-closed. Thus we get

Theorem 23. For each $n<\omega$, if it is consistent there is a huge cardinal, it is consistent that first countable weakly $\aleph_{1}$-collectionwise Hausdorff spaces are weakly $\aleph_{n}$-collectionwise Hausdorff.

Of course this does not tell us how to e.g. get both weakly $\aleph_{2}$-collectionwise Hausdorff and weakly $\aleph_{3}$-collectionwise Hausdorff simultaneously. An approach to doing that would be to consider Foreman's collapse of a 2-huge $\kappa$ to $\aleph_{1}$, $j(\kappa)$ to $\aleph_{2}, j(j(\kappa))$ to $\aleph_{3}\left[\mathrm{Fo}_{1}\right]$. The hope would then be to get to $\aleph_{3}$ from $\aleph_{1}$ via $\aleph_{2}$. We could indeed get weakly $\aleph_{3}$-collectionwise Hausdorff, since the generic 2-huge embedding is definable via $K_{\aleph_{3}} * \aleph_{3}$-closed forcing, but it is no longer clear how such forcing gets us weakly $\aleph_{2}$-collectionwise Hausdorff. 
Once we give up the idea of a single embedding doing all the work, there are two reasonable approaches: either have for each uncountable cardinal $\kappa$ an embedding taking $\kappa$ to $\kappa^{+}$, or else for each uncountable cardinal $\kappa$ an embedding taking $\aleph_{1}$ to $\kappa$. Now one rapidly sees that some restrictions are necessary, since one cannot send a limit cardinal to a successor. Thus the possibilities are to send $\kappa^{+}$to $\kappa^{++}$for all $\kappa$, or to send $\aleph_{1}$ to $\kappa^{+}$for all $\kappa$. Either approach leaves open the problem of inducting up through a limit cardinal. One can always cut down to a model without inaccessibles and so avoid the regular limit problem (assuming $\mathrm{GCH}$ ). There are standard tricks that often are applicable at singulars to carry through an induction (e.g. Shelah's singular compactness $\left.\left[B-D_{1}\right]\right)$, so one can face living with them. The difficulty that the first approach presents and the second one avoids is how to take care of successors of singulars, assuming one can deal with the singulars themselves. We have no answers here, although such problems have occasionally been successfully dealt with, e.g. [M]. Since we are interested mainly in transferring properties from $\aleph_{1}$ to all uncountable cardinals, we shall therefore specifically formulate axioms only for the second approach. It will be clear to the reader how to axiomatize the first approach and thereby attain results below $\aleph_{\omega}$. It must be admitted that the approach we take here is more risky from the point of view of consistency. Our first axiom puts saturation cum closure restrictions on the partial orders, the second drops the closed partial order. Since the particular generic set is irrelevant, it is convenient to write e.g. " $M \subseteq V^{\mathscr{P}}$ " with the obvious meaning. We use "crit $(j)$ " for the critical point of $j$.

Axiom I. For every uncountable $\kappa$, there exists a partial order $\mathscr{P}$ and a $\mathscr{P}$ name for a partial order, $\dot{\mathscr{R}}$, such that $\mathscr{P}$ is $\kappa$-centred, $1 \Vdash_{\mathscr{P}}$ “ $\dot{\mathscr{R}}$ is $\kappa^{+}$. closed", and in $V^{\mathscr{P} * \dot{\mathscr{R}}}$ there is an elementary embedding $j_{\kappa}: V \rightarrow M_{\kappa} \subseteq V^{\mathscr{P}_{*} \dot{\mathscr{R}}}$ such that $j^{\prime \prime} S \in M_{\kappa}$ for all $S \in[V]^{\kappa^{+}}, \operatorname{crit}\left(j_{\kappa}\right)=\aleph_{1}$, and $j_{\kappa}\left(\aleph_{1}\right)=\kappa^{+}$.

Axiom II. The same as Axiom I except $\mathscr{R}$ is not mentioned and $j_{\kappa}: V \rightarrow M_{\kappa} \subseteq$ $V^{\mathscr{P}}$.

Note that one cannot send $\aleph_{1}$ to a limit cardinal $\kappa$ by $\delta$-centred forcing, $\delta<\kappa$, by the same argument given before that you cannot send $\aleph_{1}$ to $\left(2^{\aleph_{1}}\right)^{+}$ by $\aleph_{1}$-centred forcing. The $\kappa$-case of Axiom $I$ is consistent from a huge, for fixed $\kappa=\mu^{+}, \mu$ regular, by [FL]. Even the simplest case of Axiom II is not known to be consistent. The assertion that there is a generic huge embedding sending $\aleph_{1}$ to $\aleph_{\omega+1}$ definable via $\aleph_{\omega}$-centred $* \aleph_{\omega+1}$-closed forcing is already strong enough to contradict the Covering Lemma, since it denies the existence of a nonreflecting stationary set of $\operatorname{cf}(\omega)$ ordinals in $\aleph_{\omega+1}$. This can be proved directly, but one can also use the method of proof of Theorem 1(d) to prove from the embedding that hereditarily $\aleph_{\omega}$-paracompact first countable normal spaces of cardinality $\leq \aleph_{\omega+1}$ are paracompact. By the Fact in Example 2, this prevents the existence of such a nonreflecting stationary set. One could as well proceed via the method of Theorem 1(c) and Example 3. See Theorem 38 below for stronger reflection of stationary sets.

Rather than stating results as holding up to the first regular limit, let us instead deal with Axioms $\mathrm{I}^{\prime}$ and II' $^{\prime}$ which result when we adjoin the hypothesis that there are no regular limits. If either of I or II is consistent with $\mathrm{GCH}$, so is $\mathrm{I}^{\prime}$ or $\mathrm{II}^{\prime}$ respectively. At present, any conceivable method for producing a model 
of I or II will probably produce one for $\mathrm{GCH}$ as well.

Let us now go through the results of Theorem 1, seeing how far they lift. Fortunately, the more interesting ones go all the way. It should be observed that some of the proofs proceed by induction on $\kappa$, but for those properties $\Phi$ such that $\Phi(\kappa)$ does not imply $\Phi$ holds below $\kappa$, we get $\Phi(\kappa)$ using the single embedding sending $\aleph_{1}$ to $\kappa$.

Theorem 24. Axiom I implies for every uncountable $\kappa$, if $T$ is a $\kappa^{+}$-tree with each $\aleph_{1}$-subtree special, then $T$ is special.

Proof. As in Theorem 1(a).

Theorem 25. Axiom I implies for every infinite $\kappa$, first countable weakly $\aleph_{1}$ collectionwise Hausdorff spaces are weakly $\kappa^{+}$-collectionwise Hausdorff.

Corollary 26. Axiom $\mathrm{I}^{\prime}$ plus $\mathrm{GCH}$ implies first countable regular weakly $\aleph_{1}$ collectionwise Hausdorff spaces are weakly collectionwise Hausdorff.

Proofs. Theorem 25 is proved using the ideas of the proof of $1(\mathrm{~b})$. The corollary follows if we can handle singular cardinals. The following lemma does the trick.

Lemma 27 [W]. If $\delta$ is a singular strong limit and $X$ is first countable, regular and weakly $\kappa$-collectionwise Hausdorff for all $\kappa<\delta$, then $X$ is weakly $\delta$ collectionwise Hausdorff.

Remark. It is admittedly not esthetically pleasing in the context of very large cardinal axioms to assume there are no inaccessible cardinals. One possibility is to assume that for regular iimit $\kappa$, there is a generic huge embedding sending $\aleph_{1}$ to $\kappa$, definable via property $K_{\kappa} * \kappa$-closed forcing. This addition to Axiom I would enable us to get first countable weakly $\aleph_{1}$-collectionwise Hausdorff spaces weakly $\kappa$-collectionwise Hausdorff for all regular $\kappa$.

Another approach to dealing with both singular and regular limits is to assume some sort of coherence on the partial orders. The idea would be that if e.g. $\mathscr{P}_{\aleph_{2}}$ determined a function $f$ with domain $\omega_{3}$, then $\mathscr{P}_{\aleph_{1}}$ should determine $f \mid \omega_{2}$. Thus, functions from limit cardinals could be obtained by piecing together coherent restrictions. I have not yet found any application of this idea so I will not pursue it further here. Work by Foreman and by Woodin on towers of ideals could be useful here.

Assuming the Covering Lemma, there is a model of $\mathrm{GCH}$ in which there is a $T_{3}$ first countable $<\aleph_{\omega_{1}}$-collectionwise Hausdorff space which is not $\aleph_{\omega_{1}}$ collectionwise Hausdorff [FS]. Fleissner [private communication] observed that that space is weakly $\aleph_{\omega_{1}}$-collectionwise Hausdorff, which can now be seen to follow from Lemma 27.

Theorem 28. Axiom I' plus GCH implies first countable, locally $\aleph_{2}$-c.c., $\aleph_{1}$ collectionwise Hausdorff spaces are collectionwise Hausdorff.

Proof. By induction, using the proof of 1(c). The next two cases of the lemma takes care of singular cardinals.

Lemma $29\left[\mathrm{~F}_{2}\right]$. Let $\delta$ be an uncountable limit cardinal. (a) If $\operatorname{cf}(\delta)=\omega$, then if $X$ is first countable and $\kappa$-collectionwise Hausdorff for all $\kappa<\delta$, then $X$ is $\delta$-collectionwise Hausdorff. (b) Assume $\mathrm{GCH}$. If $X$ is first countable, locally $\lambda$ c.c. for some $\lambda<\delta$, where $\omega<\operatorname{cf}(\delta)<\delta$, and $X$ is $\kappa$-collectionwise Hausdorff for all $\kappa<\delta$, then $X$ is $\delta$-collectionwise Hausdorff. 
Except at singular cardinals, the concept of weak collectionwise Hausdorffness had very little to do with the proof of Theorem 25 . What we showed is that if we had a collection $\mathscr{U}$ of e.g. $\aleph_{2}$ sets such that any subcollection of size $\aleph_{1}$ included a disjoint subcollection of that size, then $\mathscr{U}$ included a disjoint subcollection of size $\aleph_{2}$. The same proof could be used to step up from property $K_{\aleph_{1}}$ to $K_{\aleph_{2}}$, but by a result in [CN], that follows just from GCH. Stepping up to the successor of a singular however is unknown [CN, Problem 5.11], so the following result is of interest.

Theorem 30. (a) Axiom I implies property $K_{\aleph_{1}}$ partial orders have property $K_{\kappa^{+}}$ for all infinite $\kappa$. (b) Axiom I plus $\mathrm{GCH}$ implies precaliber $\aleph_{1}$ partial orders have precaliber $\kappa$ for all $\kappa$ such that $\operatorname{cf}(\kappa) \neq \aleph_{0}$.

Proof. The successor cases are proved in standard fashion and are left to the reader. In [CN, 5.8] it is proved that for strong limit $\lambda$, a partial order has precaliber $\lambda$ if and only if it has precaliber $\operatorname{cf}(\lambda)$. Thus, by positing that the only limit cardinals are singular strong ones, we finesse the singular case for precalibers. The regular limit case is due to $\mathrm{S}$. Todorčević and is included with his kind permission. Let $\left\{p_{\alpha}: \alpha<\kappa\right\} \subseteq P$, be given, $\mathscr{P}$ a countable chain condition partial order. By standard arguments, without loss of generality we may assume $\mathscr{P}$ is actually of form $\mathscr{B}-\{0\}, \mathscr{B}$ a Boolean algebra. For $F \in[\kappa]^{<\omega}$, let $p_{f}=\bigwedge\left\{p_{\alpha}: \alpha \in F\right\}$, if this exists. For each $\delta<\kappa, \operatorname{cf}(\delta)>\omega$, fix a maximal-under-inclusion $\mathscr{F}_{\delta} \subseteq[\delta]^{<\omega}$ such that

(1) $p_{F}>0$, for all $F \in \mathscr{F}$,

(2) $p_{F} \wedge p_{\delta}=0$, for all $F \in \mathscr{F}_{\delta}$,

(3) $F \neq F^{\prime}$ implies $p_{F} \wedge p_{F^{\prime}}=0$.

Then $\mathscr{F}_{\delta}$ is countable. Since $\kappa$ is strongly inaccessible, we can get a stationary $S \subseteq\{\delta<\kappa: \operatorname{cf}(\delta)>\omega\}$ and $\mathscr{F}$ such that $\mathscr{F}=\mathscr{F}$ for all $\delta \in S$. Claim $\left\{p_{\alpha}: \alpha \in S\right\}$ is centred. Suppose not and pick a minimal $F \in[S]^{<\omega}$ such that $p_{F}=0$. Let $\delta=\max F, \widehat{F}=F-\{\delta\}$. Then $\mathscr{F} \cup\{\widehat{F}\}$ also satisfies (1), (2), (3), contradicting the maximality of $\mathscr{F}=\mathscr{F}$.

Remark. The $[\mathrm{CN}]$ problem is easily solved (either way!) if one assumes Martin's Axiom plus not $\mathrm{CH}$. For then countable chain condition spaces-a fortiori spaces with precaliber $\aleph_{1}$-have precaliber $\kappa$ for every regular $\kappa<2^{\aleph_{0}}$, so we may take $2^{\aleph_{0}}>\aleph_{\omega+1}$. On the other hand, the Stone space of the Lebesgue measure algebra under MA plus not $\mathrm{CH}$ has precaliber $\aleph_{1}$ but not precaliber $2^{\aleph_{0}}[\mathrm{KT}]$, so we may take $2^{\aleph_{0}}=\aleph_{\omega+1}$.

Theorem $30(\mathrm{~b})$ allows us to obtain results as in $\left[\mathrm{T}_{3}\right]$ with weaker topological hypotheses:

Definition. $t(X)=\sup \{\min \{\kappa: p \in \bar{A} \subseteq X$ implies there is $B \subseteq A$ such that $p \in \bar{B},|B| \leq \kappa\}: p \in X\}$.

It follows from 3.21 of $\left[\mathrm{T}_{2}\right]$ that

Lemma 31. If $X$ is compact $T_{2}$ and $t(X)<\operatorname{cf}(d(X))$, then $X$ does not have precaliber $\operatorname{cf}(d(X))$.

Thus,

Theorem 32. Assume Axiom I plus GCH. Then if $X$ is a compact $T_{2}$ space with precaliber $\aleph_{1}$, if $\operatorname{cf}(d(X)) \neq \aleph_{0}$ then $d(X) \leq t(X)$. 
Another way to look at weak collectionwise Hausdorff and property $K$ results is to think of them as stepping up from a particular instance of the (generally false) partition relation $\aleph_{1} \rightarrow\left(\aleph_{1}\right)_{2}^{2}$ to the (equally false) $\kappa^{+} \rightarrow\left(\kappa^{+}\right)_{2}^{2}$. Another application of this idea, this time to algebra, was suggested by J. Steprāns. It is related to the sorts of questions considered in [FLM].

Theorem 33. Axiom I implies that if $G$ is a group such that every subgroup of size $\aleph_{1}$ includes an uncountable Abelian subgroup, then every subgroup of size $\kappa^{+}$includes an Abelian subgroup of size $\kappa^{+}$.

Proof. If $S$ is a subgroup of $G$ of cardinality $\kappa^{+}$, the usual argument will produce a commutative subset $T$ of $S,|T|=\kappa^{+}$. The subgroup generated by $T$ is the required subgroup.

Theorem 1(f) does not generalize interestingly, so we move on to (d) and its corollaries $(\mathrm{e})$ and $(\mathrm{g})$. As noted during the proof of $1(\mathrm{~d})$, by assuming $T_{2}$ we may avoid the use of (f) in the proof of (e). That assumption is a minor concession, especially when we are assuming possibly inconsistent large cardinal axioms! Now we need to use Axiom II'.

Theorem 34. Axiom $\mathrm{II}^{\prime}$ implies

(a) first countable, normal, hereditarily $\aleph_{1}$-paracompact $T_{2}$ spaces are paracompact,

(b) first countable spaces such that every open subspace has normal product with $\omega_{1}+1$ are paracompact,

(c) $\aleph_{1}$-collectionwise normal Moore spaces are metrizable.

Proof. By induction on $\delta$, we show $\delta$-paracompactness. It will be convenient to have as our inductive assumption, $\lambda$-paracompactness and hereditary $\lambda$-metacompactness, for $\lambda<\delta$. In contrast to Lemma 11 , Lemma 10 does not require a restriction on the cardinality of the space, so we can carry out the proof for paracompactness in the successor case of the induction just as in the proof of Theorem 1(d). In particular, note that the formulation of Axiom II assures us that $\left\{j^{\prime \prime} U_{\alpha}: \alpha<\kappa^{+}\right\} \in M_{\kappa}$, where $\mathscr{U}=\left\{U_{\alpha}\right\}_{\alpha<\kappa^{+}}$is a cover of $X$ in $V$. (This particular formulation was suggested by M. Magidor, to whom we are grateful.) By the hereditary $j\left(\aleph_{1}\right)$-paracompactness of $j(X)$ and $T_{2}$, it follows that $\mathscr{U}$ has a $\sigma$-discrete open refinement in $V^{\mathscr{P}}$ and hence a $\kappa$-disjoint one in $V$, whence as in the proof of Lemma 19 , we get the desired locally finite refinement of $\mathscr{U}$.

In the singular case, an open cover of size $\delta$ is the union of $\operatorname{cf}(\delta)$ open collections, each of size less than $\delta$. If we had assumed hereditary normality, we could inductively carry an assumption of hereditary $\lambda$-paracompactness, $\lambda<\delta$, and then the proof could proceed straightforwardly using Lemma 13, but we will avoid that assumption. Instead, note that for $\delta$ singular, $\delta$-collectionwise normality follows from having $\lambda$-collectionwise normality for all $\lambda<\delta$, which follows from the inductive assumption of $\lambda$-paracompactness. Thus we need only establish $\delta$-metacompactness. That will follow from Lemma 13 once we verify that we can inductively carry the assumption of hereditary $\lambda$-metacompactness. But we can, for if $Y \subseteq X, Y$ will be $j\left(\aleph_{1}\right)$-paracompact and hence $\kappa^{+}$screenable in $V^{\mathscr{D}}$. Therefore an open cover $\mathscr{U}=\left\{U_{\alpha}: \alpha<\kappa^{+}\right\}$of $Y$ will have a $\kappa$-disjoint refinement in $V$, whence the hereditary $\kappa$-metacompactness 
of $X$ produces a point-finite refinement by Lemma 13. That proves that the inductive assumption of hereditary $\kappa$-metacompactness yields hereditary $\kappa^{+}$metacompactness; at $\delta$-singular a purely topological argument (as in the proof of Lemma 13) shows that if $X$ is hereditarily $\lambda$-metacompact for all $\lambda<\delta$, then $X$ is hereditarily $\delta$-metacompact.

Theorem 34 enables us to obtain a version of stationary set reflection:

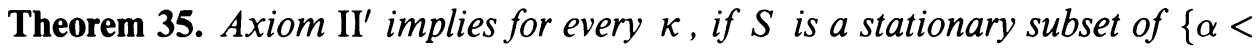
$\kappa: \operatorname{cf}(\alpha)=\omega\}$, then there is $a \mu \leq \kappa$ such that $\operatorname{cf}(\mu)=\omega_{1}$ and $S \cap \mu$ is stationary in $\mu$.

Proof. By the Fact following Example 2, $S$ with the order topology is not paracompact. $S$ is first countable and normal $T_{2}$, so $S$ is not hereditarily $\aleph_{1}$-paracompact. Therefore there is a $T \subseteq S$ and an $\alpha \in(\kappa+1)-T$ with $\operatorname{cf}(\alpha)=\omega_{1}$ such that $T \cap \alpha$ is stationary in $\alpha$. But then $S \cap \alpha$ is stationary in $\alpha$.

Theorem 35 can also be proved directly, without topology.

The reader who worked out the details of the proof of Theorem 30 (b) will have noticed that that was the only place in the paper so far where we used that the forcing is $\kappa$-centred rather than merely $\kappa$-linked. Indeed one could postulate even stronger generic embedding axioms by imposing stronger intersection properties:

Definition. A partial order $\mathscr{P}$ is $\kappa$-strongly centered if $P=\bigcup_{\alpha<\kappa} P_{\alpha}$, where for every $\alpha$ and every $S \in\left[P_{\alpha}\right]^{<\kappa}$, there is a $p \in P \leq$ each member of $S . \mathscr{P}$ is $\kappa$-dense if it has a dense set of power $\leq \kappa$.

Clearly $\kappa$-dense implies $\kappa$-strongly centred. Define Axioms $\mathrm{I}_{S}, \mathrm{II}_{S}, \mathrm{I}_{D}, \mathrm{II}_{D}$ and their primed versions in analogy to the previously defined axioms. These axioms are not much more unreasonable in the context of present-day set theory than the previous ones, but in contrast to Axiom I, they are not known to be consistent even at their first level. Although there are constructions from large cardinals that yield ideals $\mathscr{T}$ on $\omega_{1}$ such that $\left(\mathscr{P}\left(\omega_{1}\right) / \mathscr{T}\right)-\{0\}$ is $\aleph_{1}$-dense, just as the Foreman-Laver construction yields an $\mathscr{T}$ for which $\left(\mathscr{P}\left(\omega_{1}\right) / \mathscr{T}\right)-$ $\{0\}$ is $\aleph_{1}$-centred, these constructions do not apparently yield the corresponding generic huge embeddings. Ideals whose existence is considered in $\left[\mathrm{Fo}_{3}\right]$ would yield the appropriate generic huge embeddings needed for these stronger axioms. Let me give an example of how intersection properties stronger than centering can be used.

Definition [SW]. A $\sigma$-ideal $\mathscr{T}$ (we assume all ideals are proper) on a set $A$ is $(\lambda-\lambda)$-extendable if whenever $\left\{S_{\alpha}\right\}_{\alpha<\lambda}$ are subsets of $A$ which are not in $\mathscr{T}$, there is a $\sigma$-ideal $\mathscr{J} \supseteq \mathscr{T}$ and a set $L \subseteq \lambda,|L|=\lambda$, such that for each $\alpha \in L$, either $S_{\alpha}$ or $A-S_{\alpha} \in \mathscr{J}$.

Think of extending the dual filter to "measure" more sets, i.e. some sets of $\mathscr{T}$-positive measure are now assigned either $\mathscr{J}$-measure zero or $\mathscr{J}$-measure one.

Theorem 36. Con(there is a huge cardinal) implies Con(every $\left(\aleph_{1}-\aleph_{1}\right)$-extendable $\sigma$-ideal on $\aleph_{1}$ is $\left(2^{\aleph_{1}}-2^{\aleph_{1}}\right)$-extendable).

The reason I need only assume a huge rather than a new axiom, is that 
although the proof will use strong centering, it does not need the full strengih of that concept.

Definition. A partial order satisfies property $K_{\kappa, \lambda}$ (sometimes known as “( $\kappa, \kappa, \lambda)$-saturation") if given any $\kappa$ conditions, there are $\kappa$ of them such that any $\lambda$ of them have a lower bound.

Proof. Using a construction in the same genre as [ $\mathrm{K}_{1}$ and FL], Laver [L] obtained a generic huge embedding sending $\aleph_{1}$ to $\aleph_{2}$, definable via property $K_{\aleph_{2}, \aleph_{0}} * \aleph_{2}$-closed forcing; that embedding is what we shall use. Laver's model satisfies GCH, so our ideal has cardinality $\leq \aleph_{2}$. Without $2^{\aleph_{1}}=\aleph_{2}$, we could prove results about $\left(\aleph_{2}-\aleph_{2}\right)$-extendability for ideals with $\leq \aleph_{2}$ generators.

Let $\mathscr{T}$ be the $\left(\aleph_{1}-\aleph_{1}\right)$-extendable $\sigma$-ideal. Since $j$ is huge, $j^{\prime \prime} \mathscr{T} \in M$ and generates a $\sigma$-ideal which is included in the $\sigma$-ideal $j(\mathscr{T})$. Given $\left\{S_{\alpha}\right\}_{\alpha<\omega_{2}}$ in $V$, each $S_{\alpha} \subseteq \omega_{1}$, by elementarity and hugeness there is in $M$ a oneone $f: \omega_{2} \rightarrow \omega_{2} \times 2$, with projections $f_{1}$ and $f_{2}$, and a $\sigma$-ideal $\mathscr{J} \supseteq j(\mathscr{T})$ which contains $j\left(S_{f_{1}(\alpha)}\right)$ or $\omega_{2}-j\left(S_{f_{1}(\alpha)}\right)$, depending on whether $f_{2}(\alpha)=1$ or 0 . Since $\mathscr{J}$ is proper and includes $j^{\prime \prime} \mathscr{T}$, we know that in $M$ no countable union of members of $j^{\prime \prime} \mathscr{T}$ and these $j\left(S_{f_{1}(\alpha)}\right)$ or $\omega_{2}-j\left(S_{f_{1}(\alpha)}\right)$ 's equals $\omega_{2}$. By elementarity, we see in $V^{\mathscr{P} * \mathscr{\mathscr { R }}}$ that no countable union of these members of $\mathscr{T}$ and the $S_{f_{1}(\alpha)}$ or $\omega_{1}-S_{f_{1}(\alpha)}$ 's equals $\omega_{1}$. Thus we are in the familiar situation of having a function from $\omega_{2}$ into $V$ in $V^{\mathscr{P} * \mathscr{R}}$ which we then proceed to pull back. By a now standard argument, the ground model function deciding $f$ obtained by $\aleph_{2}$-closed forcing will yield a selection of $\aleph_{2}$ sets extending $\mathscr{T}$, since $f$ does. On the other hand, since $\mathscr{P}$ has property $K_{\aleph_{2}, \aleph_{0}}$, we can decide such a selector on a set of size $\aleph_{2}$ by a set of conditions such that each of its countable subsets has a lower bound. That will assure that the extension of $\mathscr{T}$ determined by the piece of the selector will be proper.

\section{APPLICATIONS OF SUPERCOMPACT CARDINALS}

We now move onto other applications. There is one case where we can successfully go from $\aleph_{1}$ to all cardinals with very little new technology. The Lévy collapse of a supercompact to $\aleph_{2}$ by countable conditions yields a model in which first countable $\aleph_{1}$-collectionwise Hausdorff spaces are collectionwise Hausdorff if they locally have density $\leq \aleph_{1}$. This is announced for local cardinality $\leq \aleph_{1}$ in $\left[S_{1}\right]$ but the same proof works. It is annoying that the greater consistency strength of hugeness enabled us to weaken the local requirement to locally $\aleph_{2}$-c.c., but did not enable us to go past $\aleph_{2}$. By combining the proof with the proof of $\left[\mathrm{S}_{1}\right]$ however, we can go "all the way" at slight cost.

Theorem 37. Let $\kappa$ be huge with $j(\kappa)$ supercompact. In the Foreman-Laver model, first countable $T_{2} \aleph_{1}$-collectionwise Hausdorff spaces are collectionwise Hausdorff if they are locally $\aleph_{2}$-c.c.

Such spaces are $\aleph_{2}$-collectionwise Hausdorff by Theorem 1(c). By GCH and elementary cardinal functions (see e.g. $[\mathrm{H}]$ ), first countable $T_{2} \aleph_{2}$-c.c. spaces have cardinality-a fortiori density $-\leq \aleph_{2}$. Recall the Foreman-Laver model is obtained by first collapsing $\kappa$ to $\aleph_{1}$ by $\mathscr{P}$, and then $j(\kappa)$ to $\aleph_{2}$ by the countably closed $\mathscr{R} \in V\left[G_{\mathscr{P}}\right]$. It can be shown that $\mathscr{P}$ has cardinality $\kappa<j(\kappa)$, and 
so $j(\kappa)$ is still supercompact in $V\left[G_{\mathscr{P}}\right]$ (see e.g. [KM] concerning "mild" forcing). By the general machinery of supercompact reflection applied to topology as e.g. in $\left[D T W_{1}\right]$, to prove that in $V\left[G_{\mathscr{P}}\right]\left[G_{\mathscr{R}}\right]$ first countable $\aleph_{2}$-collectionwise Hausdorff spaces of local density $\leq \aleph_{2}$ are collectionwise Hausdorff, it suffices to show that countably closed forcing cannot adjoin a separation of an unseparated closed discrete collection of points to such a space. In $\left[\mathrm{S}_{1}\right]$ this is shown for first countable $\aleph_{1}$-collectionwise Hausdorff spaces of local cardinality $\leq \aleph_{1}$, and the same proof works.

Our next result using supercompact cardinals applies stationary set reflection as in the conclusion of Theorem 35. Consider the principle

Stationary set reflection. For every $\kappa$, if $S$ is a stationary subset of $\{\alpha<$ $\kappa: \operatorname{cf}(\alpha)=\omega\}$, then there is an ordinal $\mu \leq \kappa$ such that $\operatorname{cf}(\mu)=\omega_{1}$ and $S \cap \mu$ is stationary in $\mu$.

We showed above that Axiom II $^{\prime}$ implies Stationary Set Reflection. An even stronger principle was shown to be consistent with GCH, modulo two supercompacts, by Shelah $\left[\mathrm{S}_{2}\right]$, and to follow from Martin's Maximum (which implies $2^{\aleph_{0}}=\aleph_{2}$ ) [FMS]:

Strong Stationary Set Reflection. For every regular $\kappa \geq \aleph_{2}$, if $S$ is a stationary subset of $\{\alpha<\kappa: \operatorname{cf}(\alpha)=\omega\}$, then there is a closed set $A$ of order-type $\omega_{1}$ included in $S$.

Definition. A subspace of a linearly ordered space is called a generalized ordered space.

(This is not the original definition, but an equivalent one.)

Theorem 38. Assume Stationary Set Reflection. Then a first countable generalized ordered space is (hereditarily) paracompact iff no closed subspace of it is homeomorphic to a stationary subset of $\omega_{1}$.

The parenthetical "hereditarily" is justified by

Lemma 39 [Lu]. Paracompact first countable generalized ordered spaces are hereditarily paracompact.

(This is stated for linearly ordered spaces [Lu] but as noted in [EL], the stronger result follows.)

The jumping off point for Theorem 38 is the following result from [EL]:

Lemma 40. A generalized ordered space is paracompact iff no closed subspace is homeomorphic to a stationary subset of a regular cardinal iff no closed subspace is homeomorphic to a stationary subset of an ordinal of uncountable cofinality.

The forward direction of the conclusion of Theorem 38 follows since a subspace of a generalized ordered space is generalized ordered. Assume then that the first countable generalized ordered space $X$ is not paracompact. Then some closed subspace $F$ of $X$ is homeomorphic via some homeomorphism $h$ to a stationary subset $S$ of a regular cardinal. Let $\kappa$ be the least such. If $\kappa=\aleph_{1}$ we are done, so suppose not. The set $F^{\prime}$ of all nonisolated points of $F$ is also closed and is homeomorphic via $h$ to the set $S^{\prime}$ of all nonisolated points of $S$. $S^{\prime}$ is also stationary in $\kappa$ (hence $F^{\prime}$ is nonempty) and, by first countability, is composed of ordinals of countable cofinality. Now apply SSR to reflect $S^{\prime}$ to 
$S^{\prime} \cap \mu, \operatorname{cf}(\mu)=\omega_{1}, S^{\prime} \cap \mu$ stationary in $\mu$. Note $S^{\prime} \cap \mu$ is a closed subset of $S^{\prime}$, for if $\alpha \in S^{\prime}$ is a limit point of $S^{\prime} \cap \mu$, there is a countable increasing sequence of members of $S^{\prime} \cap \mu$ with $\sup \alpha$. But $\operatorname{cf}(\mu)=\omega_{1}$ so $\alpha<\mu$. $S^{\prime} \cap \mu$ is not paracompact, so neither is its (closed) preimage under $h$, so that preimage has a closed subspace homeomorphic to a stationary subset of a regular cardinal $\kappa^{\prime}<\kappa$, contradiction.

Lest one be inclined to conjecture that "stationary" could be improved to "closed unbounded" in Theorem 38 we should mention that Lutzer [Lu] has an example of a nonparacompact first countable generalized ordered space of cardinality $\aleph_{1}$ which includes no topological copy of $\omega_{1}$. (This is not quite trivial since we insist on no topological copy rather than no order copy.)

Earlier we had to assume hereditary $\aleph_{1}$-paracompactness to get paracompactness in arbitrary first countable spaces. An immediate corollary of Theorem 38 is that for first countable generalized ordered spaces, it suffices to have subspaces of size $\aleph_{1}$ paracompact (in fact metalindelöf would do):

Corollary 41. Assume SSR. Then a first countable generalized ordered space is (hereditarily) paracompact iff every subspace of it of size $\aleph_{1}$ is paracompact.

This result can be extended from generalized ordered to monotonically normal spaces by some recent work of Balogh and Rudin [BR]. There is no known characterization of metrizability in generalized ordered spaces via stationary sets (but see Corollary 44 below), but there is for subspaces of ordinals:

Lemma $42[\mathrm{HJ}]$. A subspace $X$ of an ordinal is metrizable iff $X$ is first countable and for every limit ordinal $\mu$ of uncountable cofinality, $X \cap \mu$ is nonstationary in $\mu$.

From this we can prove

Corollary 43. Assume SSR. Then a subspace $X$ of an ordinal is metrizable iff $X$ is first countable and every subspace of $X$ of size $\aleph_{1}$ is metrizable.

Proof. Suppose $X$ is not metrizable. By Lemma 42 let $\nu$ be a limit ordinal of uncountable cofinality such that $X \cap \nu$ is stationary in $\nu . X \cap \nu$ is not paracompact, so it has a subspace of cardinality $\aleph_{1}$ which is not paracompact and hence is not metrizable. is:

Perhaps it is worth pointing out that an immediate corollary of Corollary 41

Corollary 44. Assume SSR. Then a locally metrizable generalized ordered space is metrizable iff every subspace of it of size $\aleph_{1}$ is metrizable.

The point is that locally metrizable spaces are first countable, and paracompact locally metrizable spaces are metrizable.

It is open whether the conclusion of Corollary 43 is consistent for arbitrary first countable spaces. In the model obtained by adding supercompact many Cohen reals, first countable spaces with every subspace of size less than continuum metrizable are metrizable [DTW 2 . Dow [Do] has proven in ZFC that compact first countable spaces are metrizable if each of their subspaces of size $\leq \aleph_{1}$ is metrizable.

It is reasonable to ask for a non-first-countable version of Theorem 38. Paracompact linearly ordered spaces are not necessarily hereditarily paracompact 
(take the Dedekind completion with endpoints of a nonparacompact linearly ordered space), so there are some differences. However, by assuming a sufficiently strong reflection principle one does get a theorem. Ben-David [B- $\left.\mathrm{D}_{2}\right]$ has shown the consistency with $\mathrm{GCH}$ of the following principle, modulo a class of supercompacts:

Full Stationary Set Reflection. For every $\kappa$, every stationary $S \subseteq \kappa$ reflects as long as $\left\{\alpha \in S: \operatorname{cf}(\alpha)^{+} \neq \kappa\right\}$ is stationary in $\kappa$.

Definition. The character of $X$,

$$
\chi(X)=\sup _{x \in X}\{\min \{\lambda: \text { there is a neighbourhood base for } x \text { of size } \leq \lambda\}\} .
$$

Theorem 45. Assume Full Stationary Set Reflection. Then a generalized ordered space $X$ with $\chi(X) \leq \lambda$ is paracompact iff no closed subspace of $X$ is homeomorphic to a stationary subset of $\lambda^{\prime}$, where $\lambda^{\prime} \leq \lambda^{+}$.

Proof. An examination of the proof of Theorem 38 reveals that all we need be assured of is that a stationary subset $S^{\prime} \subseteq\{\alpha \in \kappa: \operatorname{cf}(\alpha) \leq \lambda\}, \kappa>\lambda^{+}$, reflects to $S^{\prime} \cap \mu, \operatorname{cf}(\mu) \leq \lambda^{+}$. So long as $\operatorname{cf}(\kappa)>\lambda^{+}$, by assumption $S^{\prime}$ will reflect, so by repeated reflections we indeed do reflect to a $\mu$ with $\operatorname{cf}(\mu) \leq \lambda^{+}$.

\section{Problems}

In conclusion, let us highlight some of the more interesting problems posed by our results, in addition to the $\kappa$-centred forcing ones discussed above.

1. Prove the consistency of Axioms I or II, modulo large cardinals.

2. Prove the consistency with GCH of the existence of a partial order with precaliber $\aleph_{1}$ that does not have precaliber $\aleph_{\omega+1}$.

3. Prove the consistency of "every first countable $\aleph_{1}$-collectionwise Hausdorff space is collectionwise Hausdorff".

4. Find an example of a first countable normal $\aleph_{1}$-paracompact space that is not paracompact.

Note. T. LaBerge and A. Landver [LL] have recently proved some interesting results about (weakly) collectionwise Hausdorffness that complement ours:

Theorem. Lévy collapse a supercompact (weakly compact) cardinal to $\aleph_{2}$ with countable conditions. Then every first countable $\aleph_{1}$-collectionwise Hausdorff space is weakly $\left(\aleph_{2}\right)$-collectionwise Hausdorff.

Theorem. $\square$ implies there is a first countable $\aleph_{1}$-collectionwise Hausdorff space that is not weakly $\aleph_{2}$-collectionwise Hausdorff.

Since GCH is consistent with $\square$, this improves Example 4.

\section{REFERENCES}

[A] C. E. Aull, Some base axioms for topology involving enumerability, General Topology and its Relations to Modern Analysis and Algebra (Proc. Kanpur Topology Conf., 1968), Academia, Prague, 1971, pp. 54-61.

[BR] Z. Balogh and M. E. Rudin, Monotone normality, Topology Appl. 47 (1992), 115-127.

[B] M. Bell, On the combinatorial principle $P(c)$, Fund. Math. 114 (1981), 149-157.

[B-D ${ }_{1}$ ] S. Ben-David, On Shelah's compactness of cardinals, Israel J. Math. 31 (1978), 34-56. 
[B- $\left.\mathrm{D}_{2}\right]$ _ A Laver-type indestructibility for accessible cardinals, Logic Colloquium '86 (Hull, 1986), North-Holland, Amsterdam, 1988, pp. 9-19.

[CN] W. W. Comfort and S. Negrepontis, Chain conditions in topology, Cambridge Univ. Press, Cambridge, 1982.

[D] P. Daniels, A first countable $\omega_{1} C W H / C W H$ not $\omega_{2}$ collectionwise Hausdorff space, Q \& A General Topology 6 (1988), 129-134.

[Do] A. Dow, An empty class of nonmetric spaces, Proc. Amer. Math. Soc. 104 (1988), 999-1001.

$\left[\mathrm{DTW}_{1}\right]$ A. Dow, F. D. Tall, and W. Weiss, New proofs of the consistency of the normal Moore space conjecture I, Topology Appl. 37 (1990), 33-51.

$\left[\mathrm{DTW}_{2}\right] \ldots$ New proofs of the consistency of the normal Moore space conjecture II, Topology Appl. 37 (1990), 115-130.

[vD] E. K. van Douwen, The integers and topology, Handbook of Set-theoretic Topology (K. Kunen and J. E. Vaughan, eds.), North-Holland, Amsterdam, 1984, pp. 111-167.

[E] R. Engelking, General topology, Heldermann-Verlag, Berlin, 1989.

[EL] R. Engelking and D. Lutzer, Paracompactness in ordered spaces, Fund. Math. 94 (1977), 49-58.

[ $\left.\mathrm{F}_{1}\right]$ W. G. Fleissner, Normal Moore spaces in the constructible universe, Proc. Amer. Math. Soc. 46 (1974), 294-298.

$\left[\mathrm{F}_{2}\right]-$, Separating discrete collections of singular cardinality, Set-theoretic Topology (G. M. Reed, ed.), Academic Press, New York, 1977, pp. 135-140.

$\left[\mathrm{F}_{3}\right] \longrightarrow$, On $\lambda$ collection Hausdorff spaces, Topology Proc. 2 (1977), 445-456.

$\left[\mathrm{F}_{4}\right]$, Separation properties in Moore spaces, Fund. Math. 98 (1978), 279-286.

$\left[\mathrm{F}_{5}\right]$ - Current research on Q-sets, Colloq. Math. Soc. János Bolyai 23 (1978), 413-431.

$\left[\mathrm{F}_{6}\right] \longrightarrow$, Left-separated spaces with point-countable bases, Trans. Amer. Math. Soc. 294 (1986), 665-678.

$\left[\mathrm{Fo}_{1}\right] \mathrm{M}$. Foreman, Large cardinals and strong model theoretic transfer properties, Trans. Amer. Math. Soc. 272 (1982), 427-463.

[Fo $\left.{ }_{2}\right]$, More saturated ideals, Cabal Seminar 1979-81 (A. S. Kechris, D. A. Martin, and Y. N. Moschovakis, eds.), Lecture Notes in Math., vol. 1019, Springer-Verlag, Berlin and New York, 1983, pp. 1-27.

[Fo $\left.{ }_{3}\right]$, Potent axioms, Trans. Amer. Math. Soc. 294 (1986), 1-28.

[FL] M. Foreman and R. Laver, Some downwards transfer properties for $\aleph_{2}$, Adv. in Math. 67 (1988), 230-238.

[FLM] V. Faber, R. Laver, and R. McKenzie, Coverings of groups by Abelian subgroups, Canad. J. Math. 30 (1978), 933-945.

[FMS] M. Foreman, M. Magidor, and S. Shelah, Martin's maximum, saturated ideals, and nonregular ultrafilters. Part I, Ann. of Math. 127 (1988), 1-47.

[FS] W. G. Fleissner and S. Shelah, Collectionwise Hausdorff: incompactness at singulars, Topology Appl. 31 (1989), 101-107.

[H] R. Hodel, Cardinal functions. I, Handbook of Set-theoretic Topology (K. Kunen and J. E. Vaughan, eds.), North-Holland, Amsterdam, 1984, pp. 1-62.

[HJ] A. Hajnal and I. Juhász, On spaces in which every small subspace is metrizable, Bull. Polon. Acad. Sci. Sér. Math Astronom. Phys. 24 (1976), 727-731.

[J] I. Juhász, Cardinal functions in topology-ten years later, Math. Centre Tracts 123, NorthHolland, Amsterdam, 1980.

[K $\left.\mathrm{K}_{1}\right] \quad$ K. Kunen, Saturated ideals, J. Symbolic Logic 43 (1978), 65-76.

$\left[\mathrm{K}_{2}\right]$ - Set theory, an introduction to independence proofs, North-Holland, Amsterdam, 1980.

[KM] A. Kanamori and M. Magidor, The evolution of large cardinal axioms in set theory, Proc. Conf. on Higher Set Theory, Lecture Notes in Math., vol. 669, Springer, Berlin, 1978, pp. 99-275.

[KT] K. Kunen and F. D. Tall, Between Martin's Axiom and Souslin's Hypothesis, Fund. Math. 102 (1979), 173-181. 
[L] R. Laver, An ( $\left.\aleph_{2}, \aleph_{2}, \aleph_{0}\right)$-saturated ideal on $\aleph_{1}$, Logic Colloquium 1980 (Prague), NorthHolland, Amsterdam, 1982, pp. 173-180.

[LL] T. LaBerge and A. Landver, Reflection and weakly collectionwise Hausdorff spaces, Proc. Amer. Math. Soc. (to appear).

[Lu] D. Lutzer, Ordinals and paracompactness in ordered spaces, TOPO 72, Proc. Second Pittsburgh Topology Conf., Lecture Notes in Math., vol. 378, Springer, Berlin and New York, 1974, pp. 258-266.

[M] M. Magidor, Reflecting stationary sets, J. Symbolic Logic 47 (1982), 755-771.

[MS] E. Milner and S. Shelah, Some theorems on transversals, Infinite and Finite Sets (A. Hajnal, R. Rado, and V. T. Soś, eds.), North-Holland, Amsterdam, 1975, pp. 1115-1126.

[P] T. Przymusiński, Products of normal spaces, Handbook of Set-theoretic Topology (K. Kunen and J. E. Vaughan, eds.), North-Holland, Amsterdam, 1984, pp. 781-826.

$\left[\mathrm{S}_{1}\right] \quad S$. Shelah, Remarks on $\lambda$-collectionwise Hausdorff spaces, Topology Proc. 2 (1977), 583-592.

$\left[\mathrm{S}_{2}\right] \quad$, Proper forcing, Lecture Notes in Math., vol. 940, Springer-Verlag, Berlin and New York, 1982.

[So] L. Soukup, A nonspecial $\omega_{1}$-tree with special $\omega_{1}$-subtrees, Comment. Math. Univ. Carolin. 31 (1990), 607-612.

[SK] C. I. Steinhorn and J. H. King, The uniformization property for $\aleph_{2}$, Israel J. Math. 36 (1980), 248-256.

[SRK] R. Solovay, W. Reinhardt, and A. Kanamori, Strong axioms of infinity and elementary embeddings, Ann. Math. Logic 13 (1978), 73-116.

[SW] J. Steprāns and S. Watson, Extending ideals, Israel J. Math. 54 (1986), 201-226.

$\left[\mathrm{T}_{1}\right]$ F. D. Tall, Set-theoretic consistency results and topological problems concerning the normal Moore space conjecture and related problems, Thesis, Univ. of Wisconsin, Madison, 1969; Dissertationes Math. 148 (1977), 1-53.

$\left[\mathrm{T}_{2}\right] \longrightarrow$, The countable chain condition versus separability-applications of Martin's Axiom, General Topology Appl. 4 (1974), 315-339.

[ $\left.\mathrm{T}_{3}\right]$ - Weakly collectionwise Hausdorff spaces, Topology Proc. 1 (1976), 295-304.

$\left[\mathrm{T}_{4}\right] \ldots$, Normality versus collectionwise normality, Handbook of Set-theoretic Topology (K. Kunen and J. E. Vaughan, eds.), North-Holland, Amsterdam, 1984, pp. 685-732.

$\left[\mathrm{T}_{5}\right]$, Topological applications of supercompact and huge cardinals, General Topology and its Relations to Modern Analysis and Algebra (Proc. 6th Prague Topology Sympos., 1986), Heldermann-Verlag, Berlin, 1988, pp. 545-558.

[ $\left.\mathrm{T}_{6}\right] \ldots$, More topological applications of generic elementary embeddings, Topology Appl. 44 (1992), 353-358.

[ $\left.\mathrm{T}_{7}\right]$ - Some applications of a generalized Martin's Axiom, Topology Appl. (to appear).

$\left[\mathrm{T}_{8}\right] \ldots, \sigma$-centred forcing and reflection of $(s u b)$ metrizability, Proc. Amer. Math. Soc. (to appear).

[To] S. Todorčević, Special square sequences, Proc. Amer. Math. Soc. 105 (1989), 199-205.

[W] S. Watson, Comments on separation, Topology Proc. 14 (1989), 315-372.

Department of Mathematics, University of Toronto, Toronto, Canada M5S 1A1

E-mail address: tall@math.toronto.edu 